\title{
HOMOGENIZATION OF A SHRINKING CORE MODEL FOR GAS-SOLID REACTIONS IN GRANULAR PARTICLES*
}

\author{
BENJAMIN M. SLOMAN ${ }^{\dagger}$, COLIN P. PLEASE ${ }^{\dagger}$, AND ROBERT A. VAN GORDER ${ }^{\dagger}$
}

\begin{abstract}
Reactions between gases and solid particles are commonly modeled using a shrinking core framework, where a sharp interface between an inner unreacted core and an outer product shell moves inward until the reaction is complete. However, for some physical systems this sharp divide is not present, and so a better model is needed to capture a transition region for the reaction. We are interested in large particles made of many small grains where there are strong interactions between microscale granular and macroscale particulate effects, and where a shrinking core model represents behavior at the microscale level. We obtain homogenized equations for macroscale behavior by exploiting the small ratio of granular to particle lengthscales. These macroscale equations allow for a diffuse reaction front, as well as a sharp interface between reacted and unreacted solid material. We analyze the resulting model asymptotically in the limits where the reaction time is rate-limited by chemical kinetics, and separately by diffusion, determining the thickness of the reaction front. Numerical simulations support the law of additive reaction times, which states that the total reaction time is given as the sum of the conversion times under these limits. We further show how the model results can be extended to incorporate the transport of the product gas out of the solid particle, using a binary Fickian diffusion model. In metallurgical production the particle size and porosity are known to influence reaction times. Our results help quantify these effects and may be an aid in raw material selection.
\end{abstract}

Key words. homogenization, gas-solid reactions, porous media, shrinking core model, diffusion, asymptotics

AMS subject classifications. 35B27, 35C20, 80A30, 92E20

DOI. $10.1137 / 17 \mathrm{M} 1159634$

1. Introduction. Reactions between gases and solids occur in a wide range of chemical and engineering settings. For example, combustion of solid fuels, roasting of sulphide ores, and incineration of household waste are all listed as applications in the seminal book of Szekely, Evans, and Sohn [33]. Reactions between gases and solids are commonplace in industry, and so an improved understanding of the physical and chemical mechanisms involved is valuable. In [14], combustion of char is discussed, with solid carbon reacting with oxygen to form carbon monoxide. We developed a model of a furnace used for silicon production in [25], in which the reaction between solid carbon and gaseous silicon monoxide is a crucial part of the process of extracting silicon from quartz rock $[20,24]$.

Several modeling approaches have been utilized to understand the behavior of gas-solid reactions. Reactions between solid particles and a background, or perhaps flowing, gas will proceed differently from those of a well-mixed system, since the gas must be transported to the solid material before the reaction takes place, and then reaction products either remain in place or are transported away, influencing the reaction rate. The simplest means of capturing this interaction is the shrinking core model $[17,31]$, in which a solid particle is assumed to consist of an inner unreacted

${ }^{*}$ Received by the editors December 4, 2017; accepted for publication (in revised form) November 19, 2018; published electronically February 5, 2019.

http://www.siam.org/journals/siap/79-1/M115963.html

Funding: This work was supported by the EPSRC Centre for Doctoral Training in Industrially Focused Mathematical Modelling (EP/L015803/1) in collaboration with Elkem.

${ }^{\dagger}$ Mathematical Institute, University of Oxford, Oxford, OX2 6GG, UK (Ben.Sloman@maths. ox.ac.uk, please@maths.ox.ac.uk, Robert.VanGorder@maths.ox.ac.uk). 
core of the solid reactant surrounded by a shell of solid product. The interface between these regions moves inward, shrinking the core. This model is typically considered in a simplified geometry - either a slab, cylinder, or sphere [28]. The shrinking core model captures gas diffusion and kinetic rates as possible rate-limiting mechanisms, and allows for interactions between these effects. Shrinking core models are often used in engineering applications, for example, modeling the reaction between gaseous silicon monoxide and solid carbon particles in the context of silicon production [20, 24]. In a grain model [28, 29, 32], a solid particle is assumed to be composed of many smaller grains, each of which undergoes a shrinking core-type reaction. Careful coupling must take place between the dynamics at the microscopic grain level and at the larger particle level. The main distinction between these models is that the shrinking core has a sharp interface between reacted and unreacted regions in the solid, whereas a grain model allows for a diffuse layer on the macroscale, where the macroscale concentration of the reactant solid can vary continuously. Melchiori and Canu [18] compare these models and (with other authors) propose a single particle model including multiple reactions in a porous solid [19]. Random pore models [3, 4] allow for a diffuse reaction front in the solid while incorporating spatially varying pore sizes.

The concept of a shrinking core model has been exploited extensively in modeling of lithium batteries, as set out by Srinivasan and Newman [30]. These ideas were further developed, for example, in $[1,9,10,26]$. Such models describe how lithium in an electrode diffuses into small particles from the particle surface. The lithium changes the phase of the material, and these models account for diffusion from the particle surface to the phase change boundary. The particles are typically taken to be spherical in shape, and the boundary position between the outer lithium-rich region and the depleted inner core is tracked as part of the problem. The work presented in the present paper has a number of similarities to the shrinking core porous electrode theory of batteries, but is also different. In particular, we consider the problem of a reaction front within the small microscale grains, where one reaction species diffuses from the particle surface and the other reaction species is in the grain. Upscaling electrochemical particle models to the full battery results in the porous electrode model of Doyle, Fuller, and Newman [11], and this is similar to the homogenization procedure we undertake here.

We are interested in deriving a model for the reaction of a gas with a porous solid particle, which we assume is composed of many smaller-scale grains. This model will allow for various rate-limiting steps, including gaseous diffusion in the pores and the reaction rate in solid grains. In order to use behavior on the granular microscale to find effective equations at the macroscale particle level, we employ homogenization theory, an overview of which can be found in $[2,22]$. We use the work of Bruna and Chapman [5] and Dalwadi and colleagues $[7,8]$ as a framework for our approach, since they also consider diffusion in spatially varying porous media. In $[5,8]$, the porous geometry consists of solid cylinders and spheres, with the radius depending on the macroscale. In [7], these solid obstacles can also grow over time, due to a reaction, reducing the region in which diffusion can occur. We consider an external gas outside the particle to diffuse between the grains and then be transported into the grain to the shrinking core reaction front, where it reacts with the solid core to create a new solid. We first assume that any product gas has no influence on the reaction, and we later show that the models and analysis can easily be extended to include product gas effects.

The paper is organized as follows. In section 2 we present a mathematical formulation of the canonical shrinking core model, which assumes an inward-moving 
sharp interface between an unreacted solid core and a reacted solid outer shell. In section 3 we use this shrinking core model as a basis for behavior at the microscale granular level. We take $\delta$ to be the ratio of micro- to macroscale lengthscales; in the limit $\delta \rightarrow 0$ we obtain a system of homogenized equations that captures behavior on the macroscale. Two distinguished limits arise depending on the ratio of the gas diffusivity in the grain to that in the pore space. The model arising from the more physically relevant distinguished limit is analyzed in section 5 , with numerical simulations and asymptotics discussed in the cases where the process is diffusion-controlled or reaction-controlled. In section 6 we show how the model development and analysis can be extended to include the dynamics of a product gas. Comparison with results present in the literature are given in section 7 , with conclusions drawn in section 8 .

\section{Shrinking core model.}

2.1. Physical derivation. We are interested in the irreversible chemical reaction between a gas $A$ and solid $B$, which forms the products of gas $F$ and solid $H$,

$$
A_{\text {gas }}+\sigma_{1} B_{\text {solid }} \rightarrow \sigma_{2} F_{\text {gas }}+\sigma_{3} H_{\text {solid }}
$$

where $\sigma_{1}, \sigma_{2}$, and $\sigma_{3}$ are stoichiometric coefficients. We assume that these species are in an idealized spherical particle consisting of a central spherical core of nonporous solid $B$, surrounded by a shell of porous solid $H$, with the reaction taking place at the interface between $B$ and $H$. We further assume that when species $B$ reacts with the gas it creates a solid material of constant porosity $\psi$. For simplicity, no volumetric changes are modeled, so that when the reaction is complete the spherical particle of solid $H$ is the same size as the initial spherical particle of solid $B$. We assume that both gases coexist in the outer shell, but that no gas is present inside the inner core. We are interested in spherically symmetric behavior only, and assume that the particle is of radius $r=R_{p}(\mathrm{~m})$, where $r$ is our coordinate. The interface between the inner core and the outer shell is given by $r=l(t)(\mathrm{m})$. Thus there is a sharp divide between the reacted and unreacted regions. A schematic of this is given in Figure 1, where $P_{A}$ and $P_{F}$ (both $\mathrm{kg} / \mathrm{ms}^{2}$ ) are the partial pressures of gases $A$ and $F$, respectively, with external atmospheric partial pressures given by $P_{A 0}(t)$ and $P_{F 0}(t)$.

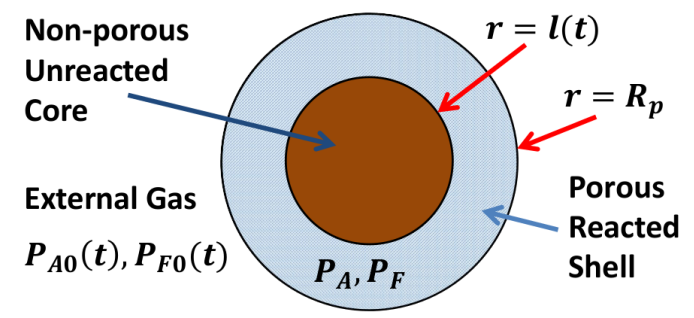

FIG. 1. Schematic of the shrinking core model, showing the fixed outside radius $r=R_{p}$ and the moving internal radius $r=l(t)$ of a spherical shell.

For simplicity, we first consider only the reactant gas, and thus assume that $P_{F}$ has no influence on the dynamics of the reaction. Later, in section 6 , we show that the analysis in sections $2-5$ can easily be extended to incorporate the diffusional hindrance of the product gas.

We let the absolute density of $A$ be given by $\rho_{A}\left(\mathrm{~kg} / \mathrm{m}^{3}\right)$, with the effective density given by $\psi \rho_{A}$. We assume that gas $A$ will be transported by diffusion from 
the external atmosphere at $r=R_{p}$ to the interface $r=l(t)$, then react with the solid $B$ to form gas $F$ and solid $H$, according to reaction (1). Diffusive transport of $A$ implies we have the following equation arising from conservation of mass:

$$
\frac{\partial\left(\psi \rho_{A}\right)}{\partial t}=D \nabla^{2}\left(\psi \rho_{A}\right), \quad l(t) \leq r \leq R_{p}
$$

where we assume constant diffusivity $D\left(\mathrm{~m}^{2} / \mathrm{s}\right)$.

We can relate $P_{A}$ and $\rho_{A}$ through the ideal gas law

$$
P_{A}=\frac{\rho_{A}}{M_{A}} R_{g} T
$$

where we have used $R_{g}$ to denote the gas constant $(8.314 \mathrm{~J} / \mathrm{molK}) . M_{A}(\mathrm{~kg} / \mathrm{mol})$ is the molar mass of $A$, and $T(\mathrm{~K})$ is the temperature. Thus, assuming that the particle is isothermal, and noting that porosity is constant, we substitute (3) into (2) to obtain the diffusion equation for pressure:

$$
\frac{\partial P_{A}}{\partial t}=D \nabla^{2} P_{A}, \quad l(t) \leq r \leq R_{p}
$$

At the external boundary the known external pressure implies

$$
P_{A}=P_{A 0}(t) \text { at } r=R_{p} .
$$

We let $K_{A}, K_{B}\left(\mathrm{~mol} / \mathrm{m}^{2} \mathrm{~s}\right)$ be the rates at which $A$ and $B$ are consumed, per unit time per unit surface area of the unreacted core. By the stoichiometry in (1) we have

$$
K_{A}=K_{B} / \sigma_{1} .
$$

For simplicity, we assume that reaction (1) follows a first order model, so that

$$
K_{A}=k P_{A} B_{0},
$$

where $B_{0}\left(\mathrm{~mol} / \mathrm{m}^{3}\right)$ is the assumed constant local concentration of $B$ in the unreacted core, and $k\left(\mathrm{~m}^{2} \mathrm{~s} / \mathrm{kg}\right)$ is the reaction constant. $B_{0}$ is given in terms of the molar mass $M_{B}(\mathrm{~kg} / \mathrm{mol})$ and absolute mass density $\rho_{B}$ by $B_{0}=\rho_{B} / M_{B}$. An Arrhenius law or higher order kinetic rates could be used if these are more appropriate for the particular application. Melchiori and colleagues $[18,19]$ use a general power $P_{A}^{\alpha}$ in their continuum model, and we comment on this in section 7.4. At the moving reaction interface $r=l(t)$, we have the following conditions, which have been determined by considering the mass flux relative to the interface for $A$ and then $B$ and using (3):

$$
\begin{gathered}
-\frac{D}{R_{g} T} \frac{\partial P_{A}}{\partial r}+\frac{P_{A}}{R_{g} T} \frac{d l}{d t}=-K_{A} \text { at } r=l(t), \\
\frac{\rho_{B}}{M_{B}} \frac{d l}{d t}=-K_{B} \text { at } r=l(t) .
\end{gathered}
$$

Note that $l(t) \geq 0$, so that strictly speaking the right-hand side of (9) should be $-K_{B} \mathbb{1}_{l(t) \geq 0}$, where $\mathbb{1}_{l(t) \geq 0}$ is the indicator function, so that the interface stops moving once it reaches $l(t)=0$. However, from now on we will take this as assumed for brevity of notation. We note that, by (6), the right-hand side of (9) can be written as $-\sigma_{1} K_{A}$. 
2.2. Dimensionless model. To analyze the problem, we now nondimensionalize using the scalings

$$
t=\tau_{s} \bar{t}, \quad r=R_{p} \bar{r}, \quad l=R \bar{l}, \quad P_{A}=P_{\mathrm{TOT}} \bar{P}_{A}, \quad P_{A 0}=P_{\mathrm{TOT}} \bar{P}_{A 0},
$$

where $P_{\mathrm{TOT}}$ is a constant reference pressure. We introduce three dimensionless parameters, namely,

$$
\hat{\epsilon}=\frac{R_{p}^{2}}{D \tau_{s}}, \quad \hat{\beta}=\frac{R_{g} T k B_{0} R_{p}}{D}, \quad \hat{\gamma}=\frac{\sigma_{1} k P_{\mathrm{TOT}} \tau_{s}}{R_{p}},
$$

where the hat notation is to avoid confusion with parameters introduced later in (29) and is used from section 3 onward. Dropping the overbar notation from the variables in (10), we obtain the system

$$
\begin{gathered}
\hat{\epsilon} \frac{\partial P_{A}}{\partial t}=\nabla^{2} P_{A}, \quad l(t) \leq r \leq 1, \\
P_{A}=P_{A 0}(t) \text { at } r=1, \\
-\frac{\partial P_{A}}{\partial r}+\hat{\epsilon} P_{A} \frac{d l}{d t}=-\hat{\beta} P_{A} \text { at } r=l(t), \\
\frac{d l}{d t}=-\hat{\gamma} P_{A} \text { at } r=l(t) .
\end{gathered}
$$

We now discuss some of the behavior in limiting cases of the dimensionless parameters. The parameter $\hat{\epsilon}$ measures the ratio of the timescale for diffusion within the particle to the timescale for the particle to completely react. Thus, small $\hat{\epsilon}$ means that gas diffuses throughout the particle quickly compared to the particle reaction time, and in particular we do not need to consider the change in the total amount of gas in the particle from the reaction. We are free to choose the timescale $\tau_{s}$, and so could set any of the above parameters to unity with an appropriate choice, if desired. However, we will take $\tau_{s}$ to be an experimentally found time (in the setting of interest) for a typical particle to completely react. Typically, such a timescale allows a simplification of our system by using the quasi-steady state assumption (QSSA), sometimes called the pseudo-steady state assumption [34], corresponding to taking the limit $\hat{\epsilon} \rightarrow 0$. $\mathrm{Xu}$, Sun, and Khaleel [35] compare results to solutions found without using a quasisteady state, with good agreement when the concentration of the reactant gas is low compared to that of the product solid. We use the quasi-steady limit from now on in this paper.

The parameter $\hat{\beta}$ is a measure of the ratio of the amount of gas $A$ lost from the reaction to the amount of gas transported to the interface by the diffusive flux. Thus, if $\hat{\beta}$ is large, the reaction is quick compared to the time for gas to get to the interface, and diffusion through the reacted material is the process that determines the overall reaction time. If, however, $\hat{\beta}$ is small, then the chemical reaction time is slower than the diffusive flux, and hence the process is reaction-limited. The parameter $\hat{\gamma}$ is a measure of how far the interface moves for a given amount of the gas-solid reaction. 
2.3. Quasi-steady limit. We now study the system (12)-(15) in the limit $\hat{\epsilon} \rightarrow$ 0 . This allows us to neglect the left-hand side of (12), and the second term in (14), to give the canonical shrinking core problem that has been studied extensively in the literature $[28,31,33]$. We find $P_{A}$ by integrating (12), to obtain

$$
r^{2} \frac{\partial P_{A}}{\partial r}=A(t), \quad l(t) \leq r \leq 1,
$$

where, using (14), we have introduced

$$
A(t)=\hat{\beta} l(t)^{2} P(l(t), t) .
$$

Integrating (16) and using (13), we find

$$
P_{A}(r, t)=P_{A 0}(t)\left[1-\frac{\hat{\beta} l(t)^{2}}{1+\hat{\beta} l(t)(1-l(t))}\left(\frac{1}{r}-1\right)\right], \quad A(t)=P_{A 0}(t) \frac{\hat{\beta} l(t)^{2}}{1+\hat{\beta} l(t)(1-l(t))} .
$$

Substituting $P_{A}$ from (18) into (15), we then obtain the ODE for the $l(t)$ problem:

$$
\frac{d l}{d t}=-\hat{\gamma} \frac{P_{A 0}(t)}{1+\hat{\beta} l(t)(1-l(t))}, \quad l(0)=1 .
$$

Integrating (19), we find

$$
\hat{\beta} \frac{l(t)^{3}}{3}-\hat{\beta} \frac{l(t)^{2}}{2}-l(t)+1+\frac{\hat{\beta}}{6}=\hat{\gamma} \int_{u=0}^{t} P_{A 0}(u) d u .
$$

Given the external pressure $P_{A 0}(t),(20)$ gives a cubic which can be solved to determine the position of the interface $l(t)$, upon taking the monotonically decreasing real root which starts at $l(0)=1$. In particular, we can find the time $t=t^{*}$ when the particle is first fully reacted (that is $l\left(t^{*}\right)=0$ ), given by the integral condition

$$
\int_{u=0}^{t^{*}} P_{A 0}(u) d u=\left(1+\frac{\hat{\beta}}{6}\right) \frac{1}{\hat{\gamma}}
$$

and hence for finite $\hat{\beta}$ and nonzero $\hat{\gamma}$, the particle will always be fully reacted in finite time, as long as the external pressure is nonzero. This is the classic shrinking core model, and we comment further on limits of this simple model in section 7 .

The shrinking core assumption, that there is a sharp interface between reacted and unreacted regions, is not always valid, especially for very porous particles where highly diffusive gas may access the inner part of the particle before the outer part has fully converted. This is particularly true for agglomerated solids, where small grains of the reactant solid are compressed together and held in place by a binder. A need to capture interactions between smaller granular and larger particle scales leads us to utilize homogenization theory.

3. Homogenization of a granular particle. In section 2 we considered a reacting solid particle which has an inward-moving sharp interface between a central unreacted core and a reacted shell. We now consider the reaction of a particle that is composed of many smaller grains, so there may be a diffuse region over which the reaction occurs, instead of a sharp divide. Note that grain models [33] and continuum 
models $[18,19]$ have been formed to represent such a situation. However, we use the mathematical technique of homogenization to systematically derive models, which can then be compared to those previously studied. For ease of notation, from now on we refer to the partial pressures $P_{A}$ and $P_{F}$ as $P$ and $Q$, for gases $A$ and $F$, respectively, although we will not use $Q$ until section 6 .

We follow the methodology of Bruna and Chapman [5], and Dalwadi and colleagues $[7,8]$, due to the similar nature of their problems. However, we have diffusion inside solid grains, as well as between them, so we discuss the homogenization in detail. We consider a periodic microscale consisting of three regions. A solid unreacted core occupies the region $\omega_{C}$, which is surrounded by a porous shell of the solid product $\omega_{S}$. This is, in turn, surrounded by void space $\omega_{V}$, which is filled by gas. The interface between $\omega_{V}$ and $\omega_{S}$ is denoted by $\partial \omega_{a}$, and the interface between $\omega_{S}$ and $\omega_{C}$ is denoted by $\partial \omega_{b}$. We suppose that the ratio of the microscale to macroscale lengthscales is given by $\delta \ll 1$. We allow gas to be present in $\omega_{V}$ and inside the porous matrix in $\omega_{S}$ (which has fixed porosity), but not to be present inside $\omega_{C}$. We are interested in the reactant gas pressures inside $\omega_{V}$ and $\omega_{S}$, denoted by $P^{V}$ and $P^{S}$, respectively. A schematic of the geometry is given in Figure 2, with a cubic packing of microscale grains used for simplicity. We follow the approach of Bruna and Chapman [5] to perform a formal homogenization. We let $\mathbf{x}$ be the macroscale variable and introduce $\mathbf{y}=\mathbf{x} / \delta$ as the microscale variable, treating $\mathbf{x}$ and $\mathbf{y}$ as independent.

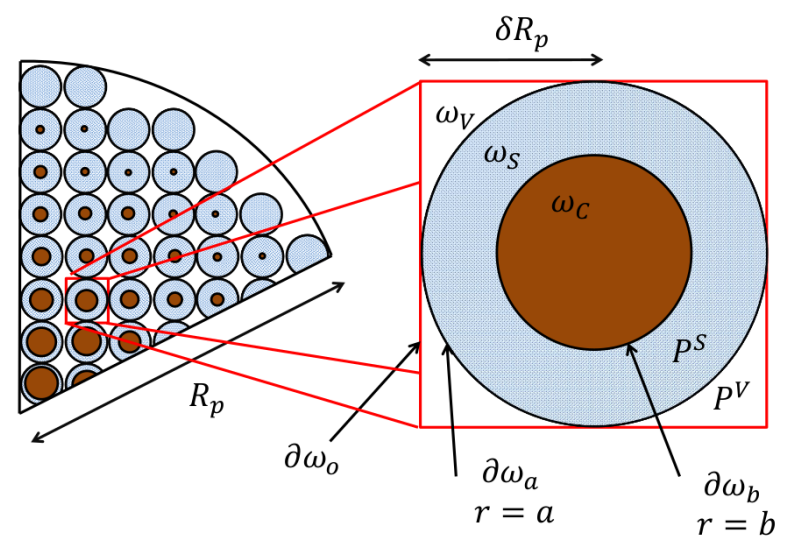

FIG. 2. Schematic of the problem geometry, with macroscale in the left wedge and microscale in the right square. Note that this is a two-dimensional plane view of the cubic packing, whereas the problem we are concerned with is three-dimensional.

We consider the scalings

$$
P^{V}=P_{\mathrm{TOT}} \hat{P^{V}}, \quad P^{S}=P_{\mathrm{TOT}} \hat{P^{S}}, \quad t=\tau_{s} \hat{t}, \quad \mathbf{x}=R_{p} \hat{\mathbf{x}}, \quad \mathbf{y}=\delta R_{p} \hat{\mathbf{y}} .
$$

We assume that the interfaces $\partial \omega_{a}$ and $\partial \omega_{b}$ are spheres with centers at the origin of each cell, with radii slowly varying functions of center position. We allow the position of $\partial \omega_{b}$ to vary with time. We thus let $b(\mathbf{x}, t)=\mathrm{B}(\mathbf{x}, t) / \delta$ be the radii of the unreacted grains of solid $B$, and $a(\mathbf{x})=\mathrm{A}(\mathbf{x}) / \delta$ the outer radius of the shells of the solid product $H$, where lowercase $a, b$ denote variables on the microscale, and uppercase A, B denote macroscale variables. For simplicity we treat the outer shell radii as constant, so that $a(\mathbf{x}) \equiv a$.

We take the diffusivity in the void $D_{V}\left(\mathrm{~m}^{2} / \mathrm{s}\right)$ to be a typical dimensional scale, but note that the ratio with the diffusivity in the product shell, $D_{S}\left(\mathrm{~m}^{2} / \mathrm{s}\right)$, will 
depend on the specific reaction of interest. We allow for different limits of $D_{S} / D_{V}$ to be considered by writing

$$
\frac{D_{S}}{D_{V}}=\delta^{n} \nu
$$

for $\nu$ an $\mathcal{O}(1)$ parameter and $n \in \mathbb{R}$. Typically, we would physically expect $n>0$, that is, $D_{S}<D_{V}$, since it is harder for gas to diffuse through the porous matrix than through the void space. However, grains may be held together by a binder which fills the void space, and so it is possible to have $n<0$, that is, $D_{V}<D_{S}$. In this section, we present the two-scale problem using a general $n$, then consider how the choice of $n$ leads to different homogenized models in section 4.

We find the field equations for $\hat{P}^{V}$ and $\hat{P}^{S}$ by using a similar process to that described in section 2. Upon dropping the hat notation, we obtain

$$
\begin{gathered}
\epsilon \frac{\partial P^{V}}{\partial t}=\nabla^{2} P^{V}, \quad \mathbf{y} \in \omega_{V}, \\
\frac{\epsilon}{\nu \delta^{n}} \frac{\partial P^{S}}{\partial t}=\nabla^{2} P^{S}, \quad \mathbf{y} \in \omega_{S} .
\end{gathered}
$$

We have continuity of pressure and flux at the interface $\partial \omega_{a}$,

$$
P^{V}=P^{S}, \quad \nabla P^{V}=\delta^{n} \nu \nabla P^{S}, \quad \mathbf{y} \in \partial \omega_{a} .
$$

In order to employ homogenization, we enforce that $P^{V}$ is periodic in $\mathbf{y}$. We also have the conditions for the interface $\partial \omega_{b}$, which are analogous to (14) and (15),

$$
\begin{gathered}
-\delta^{n} \nu \nabla P^{S} \cdot \mathbf{n}+\epsilon P^{S} \frac{\partial b}{\partial t}=-\delta \beta P^{S}, \quad \mathbf{y} \in \partial \omega_{b}, \\
\frac{\partial b}{\partial t}=-\gamma P^{S}, \quad \mathbf{y} \in \partial \omega_{b},
\end{gathered}
$$

where (28) is only defined for $b(t) \geq 0$, so that when $b(t)$ is first zero it remains in that state for all subsequent time. In addition to $\delta$ and $\nu$, we also have the dimensionless parameters $\epsilon, \beta$, and $\gamma$. These are given by

$$
\epsilon=\frac{R_{p}^{2}}{D_{V} \tau_{s}}, \quad \beta=\frac{R_{g} T k_{m} B_{0} R_{p}}{\delta D_{V}}, \quad \gamma=\frac{\sigma_{1} k P_{\mathrm{TOT}} \tau_{s}}{R_{p}},
$$

which are the same as the hatted parameters in (11), except with $D_{V}$ replacing $D$ and $k_{m} / \delta$ replacing $k . k_{m}\left(\mathrm{~m}^{2} \mathrm{~s} / \mathrm{kg}\right)$ is the microscale reaction constant and is such that $k_{m} / k=\mathcal{O}(\delta)$. As is similarly argued in [8], there are an $\mathcal{O}\left(1 / \delta^{3}\right)$ number of microscale spherical grains in the macroscale particle, each with surface area $\mathcal{O}\left(\delta^{2}\right)$. Thus the overall surface area for the reaction is $\mathcal{O}(1 / \delta)$. Hence for an $\mathcal{O}(1)$ overall reaction rate we need the reaction at the microscale interface to be $\mathcal{O}(\delta)$, and the right-hand side of (27) is accordingly that scale. We let $\beta$ and $\gamma$ be order one parameters, but treat $\epsilon$ as sufficiently small that it does not enter the analysis, so that $\epsilon=\mathcal{O}\left(\delta^{n}\right)$.

We note that the gradient operator should now incorporate both the macro- and microscales, and so have

$$
\nabla \rightarrow \nabla_{\mathbf{x}}+\frac{1}{\delta} \nabla_{\mathbf{y}}
$$

Copyright $@$ by SIAM. Unauthorized reproduction of this article is prohibited. 
Similarly, using [8], the unit normal vector at $r=b$ is given by

$$
\mathbf{n}=\frac{\mathbf{n}_{\mathbf{y}}-\delta \nabla_{\mathbf{x}} b(\mathbf{x}, t)}{\left\|\mathbf{n}_{\mathbf{y}}-\delta \nabla_{\mathbf{x}} b(\mathbf{x}, t)\right\|}
$$

where we have defined $\mathbf{n}_{\mathbf{y}}:=\mathbf{y} /\|\mathbf{y}\|$ as the outward-pointing unit normal in $\mathbf{y}$ from the unreacted solid core into the porous solid matrix of the product.

Upon taking $\epsilon$ to be suitably small, the field equations are

$$
\begin{gathered}
0=\nabla_{\mathbf{y}}^{2} P^{V}+\delta\left(\nabla_{\mathbf{x}} \cdot \nabla_{\mathbf{y}} P^{V}+\nabla_{\mathbf{y}} \cdot \nabla_{\mathbf{x}} P^{V}\right)+\delta^{2} \nabla_{\mathbf{x}}^{2} P^{V}, \quad \mathbf{y} \in \omega_{V}, \\
0=\nabla_{\mathbf{y}}^{2} P^{S}+\delta\left(\nabla_{\mathbf{x}} \cdot \nabla_{\mathbf{y}} P^{S}+\nabla_{\mathbf{y}} \cdot \nabla_{\mathbf{x}} P^{S}\right)+\delta^{2} \nabla_{\mathbf{x}}^{2} P^{S}, \quad \mathbf{y} \in \omega_{S} .
\end{gathered}
$$

Letting $\mathbf{e}_{r}$ be the radial unit vector, then the conditions in (26) become

$$
P^{V}=P^{S}, \quad \nabla_{\mathbf{y}} P^{V}+\delta \nabla_{\mathbf{x}} P^{V}=\delta^{n} \nu \nabla_{\mathbf{y}} P^{S}+\delta^{n+1} \nu \nabla_{\mathbf{x}} P^{S} \quad \text { at } \mathbf{y}=a \mathbf{e}_{r} .
$$

At the interface $\mathbf{y}=b(\mathbf{x}, t) \mathbf{e}_{r}$ we have that (27) and (28) become

$$
\begin{aligned}
-\nabla_{\mathbf{y}} P^{S} \cdot \mathbf{n}_{\mathbf{y}}+\delta\left(\nabla_{\mathbf{y}} P^{S} \cdot \nabla_{\mathbf{x}} b(\mathbf{x}, t)\right. & \left.-\nabla_{\mathbf{x}} P^{S} \cdot \mathbf{n}_{\mathbf{y}}\right)+\delta^{2} \nabla_{\mathbf{x}} P^{S} \cdot \nabla_{\mathbf{x}} b(\mathbf{x}, t) \\
& =-\delta^{2-n} \frac{\beta}{\nu} P^{S}\left\|\mathbf{n}_{\mathbf{y}}-\delta \nabla_{x} b(\mathbf{x}, t)\right\| \quad \text { at } \mathbf{y}=b(\mathbf{x}, t) \mathbf{e}_{r}, \\
& \frac{\partial b(\mathbf{x}, t)}{\partial t}
\end{aligned}
$$

with the initial condition $b(\mathbf{x}, t)=a$.

4. Distinguished limits. We now examine the system (32)-(36), considering different limits of $D_{S} / D_{V}=\delta^{n} \nu$ in turn. For each distinguished limit we shall expand the pressures in terms of $\delta$, so that $P^{V}=P_{0}^{V}+\delta P_{1}^{V}+\delta^{2} P_{2}^{V}+\mathcal{O}\left(\delta^{3}\right)$ and $P^{V}=P_{0}^{V}+\delta P_{1}^{V}+\delta^{2} P_{2}^{V}+\mathcal{O}\left(\delta^{3}\right)$. We first consider the limit $n=2$ and give detailed calculations explaining the derivation of the resulting homogenized model. We then discuss the limits $n=1$ and $n=0$, before commenting on other possible limits of the ratio of diffusivities.

4.1. The $\boldsymbol{D}_{S} / \boldsymbol{D}_{V}=\boldsymbol{\delta}^{2} \boldsymbol{\nu}$ limit. We begin by examining the limit $D_{S} / D_{V}=$ $\delta^{2} \nu$, so that $n=2$. This limit is equivalent to that required to obtain the porous electrode model, as outlined by Srinivasan and Newman in [30]. In this limit we find that the $\mathcal{O}(1)$ equations give that $P_{0}^{V}$ is independent of $\mathbf{y}$, while we also have that $P_{0}^{S}$ is given by the solution of

$$
\begin{gathered}
\nabla_{\mathbf{y}}^{2} P_{0}^{S}=0, \quad \mathbf{y} \in \omega_{S}, \\
P_{0}^{S}(a, \mathbf{x}, t)=P_{0}^{V}(a, \mathbf{x}, t), \quad \mathbf{n}_{\mathbf{y}} \cdot \nabla_{\mathbf{y}} P_{0}^{S}(b(\mathbf{x}, t), \mathbf{x}, t)=\frac{\beta}{\nu} P_{0}^{S}(b(\mathbf{x}, t), \mathbf{x}, t) .
\end{gathered}
$$

In the spherical geometry we employ, the problem (37) and (38) has the solution

$$
P_{0}^{S}=\left(\frac{\frac{1}{r}-\frac{1}{a}}{\frac{1}{a}-\frac{1}{b}-\frac{\nu}{\beta b^{2}}}+1\right) P_{0}^{V},
$$

Copyright (c) by SIAM. Unauthorized reproduction of this article is prohibited. 
and, in particular, at $r=b$ we have

$$
P_{0}^{S}(b(\mathbf{x}, t), \mathbf{x}, t)=\frac{1}{1+\frac{\beta}{\nu} b\left(1-\frac{b}{a}\right)} P_{0}^{V} .
$$

At $\mathcal{O}(\delta)$, we have the system

$$
\begin{gathered}
\nabla_{\mathbf{y}}^{2} P_{1}^{V}=0, \quad \mathbf{y} \in \omega_{V}, \\
\nabla_{\mathbf{y}}^{2} P_{1}^{S}+\nabla_{\mathbf{x}} \cdot \nabla_{\mathbf{y}} P_{0}^{S}+\nabla_{\mathbf{y}} \cdot \nabla_{\mathbf{x}} P_{0}^{S}=0, \quad \mathbf{y} \in \omega_{S}, \\
P_{1}^{V}=P_{1}^{S}, \quad \nabla_{\mathbf{y}} P_{1}^{V}+\nabla_{\mathbf{x}} P_{0}^{V}=0 \quad \text { at } r=a, \\
-\left(\nabla_{\mathbf{y}} P_{1}^{S}+\nabla_{\mathbf{x}} P_{0}^{S}\right) \cdot \mathbf{n}_{\mathbf{y}}=-\frac{\beta}{\nu} P_{1}^{S} \text { at } r=b(\mathbf{x}, t),
\end{gathered}
$$

and that $P_{1}^{V}$ is periodic in $\mathbf{y}$. Note that $\mathbf{n}_{\mathbf{y}}$ points outward away from the origin. If $\mathbf{n}_{\mathbf{y}}$ and $\nabla_{\mathbf{x}} b$ are parallel, then an additional term is present on the right-hand side of (44). Thus, if $\mathbf{n}_{\mathbf{y}}=\mathbf{e}_{r}$ and $\nabla_{\mathbf{x}} b=\frac{\partial b}{\partial R} \mathbf{e}_{r}$, then the right-hand side is given by $-\frac{\beta}{\nu} P_{1}^{S}+\frac{\beta}{\nu} \frac{\partial b}{\partial R} P_{0}^{S}$. Using the standard homogenization method $[2,22]$ and the notation employed in [5], we can write the solution for $P_{1}^{V}$ as

$$
P_{1}^{V}(\mathbf{x}, \mathbf{y}, t)=-\nabla_{\mathbf{x}} P_{0}^{V}(\mathbf{x}, t) \cdot \boldsymbol{\Gamma}(\mathbf{x}, \mathbf{y}, t),
$$

where components $\Gamma_{i}$ of the vector function $\boldsymbol{\Gamma}(\mathbf{x}, \mathbf{y}, t)$ satisfy

$$
\begin{gathered}
\nabla_{\mathbf{y}}^{2} \Gamma_{i}=0, \quad \mathbf{y} \in \omega_{V}, \\
\nabla_{\mathbf{y}} \Gamma_{i} \cdot \mathbf{e}_{\mathbf{r}}=n_{y, i} \text { at } r=a,
\end{gathered}
$$

$\Gamma_{i}$ periodic in $\mathbf{y}$,

where $n_{y, i}$ is the $i$ th component of the unit vector $\mathbf{n}_{\mathbf{y}}$. Since we have a spherical boundary, the appropriate unit vector is given by $\mathbf{n}_{\mathbf{y}}=\mathbf{e}_{r}$.

To find the necessary solvability condition, we must consider the next order system. At $\mathcal{O}\left(\delta^{2}\right)$, the equations for $P_{2}^{V}$ in $\omega_{V}$ are

$$
\begin{gathered}
\nabla_{\mathbf{y}}^{2} P_{2}^{V}+\nabla_{\mathbf{x}} \cdot \nabla_{\mathbf{y}} P_{1}^{V}+\nabla_{\mathbf{y}} \cdot \nabla_{\mathbf{x}} P_{1}^{V}+\nabla_{\mathbf{x}}^{2} P_{0}^{V}=0, \quad \mathbf{y} \in \omega_{V} \\
\nabla_{\mathbf{y}} P_{2}^{V}+\nabla_{\mathbf{x}} P_{1}^{V}=\nu \nabla_{\mathbf{y}} P_{0}^{S} \text { at } \mathbf{y}=a \mathbf{e}_{r}
\end{gathered}
$$

and $P_{2}^{V}$ is periodic in $\mathbf{y}$.

We integrate (49) over $\omega_{V}$, using the divergence theorem and the boundary conditions of periodicity and (50), to obtain

$$
-\nu \int_{r=a} \nabla_{\mathbf{y}} P_{0}^{S} \cdot \mathbf{n}_{\mathbf{y}} d S_{\mathbf{y}}+\nabla_{\mathbf{x}} \cdot \int_{\omega_{V}} \nabla_{\mathbf{y}} P_{1}^{V}+\nabla_{\mathbf{x}} P_{0}^{V} d \mathbf{y}=0 .
$$

Copyright $@$ by SIAM. Unauthorized reproduction of this article is prohibited. 
Now considering $\omega_{S}$, we note, using the divergence theorem, that

$$
\nu \int_{\omega_{V}} \nabla_{\mathbf{y}} \cdot \nabla_{\mathbf{y}} P_{0}^{S} d \mathbf{y}=\nu \int_{r=a} \nabla_{\mathbf{y}} P_{0}^{S} \cdot \mathbf{n}_{\mathbf{y}} d S_{\mathbf{y}}-\nu \int_{r=b} \nabla_{\mathbf{y}} P_{0}^{S} \cdot \mathbf{n}_{\mathbf{y}} d S_{\mathbf{y}} .
$$

The integrand on the left-hand side is zero, since $P_{0}^{S}$ satisfies Laplace's equation. We can substitute the second boundary condition in (38) into the last integral, to note

$$
\nu \int_{r=a} \nabla_{\mathbf{y}} P_{0}^{S} \cdot \mathbf{n}_{\mathbf{y}} d S_{\mathbf{y}}=\beta P_{0}^{S} \int_{r=b} d S \mathbf{y} .
$$

Hence, we can rewrite (51) as

$$
\nabla_{\mathbf{x}} \cdot \int_{\omega_{V}} \nabla_{\mathbf{y}} P_{1}^{V}+\nabla_{\mathbf{x}} P_{0}^{V} d \mathbf{y}=\beta P_{0}^{S} \int_{r=b} d S \mathbf{y}
$$

The integral over the surface of the sphere of radius $r=b$ is $4 \pi b^{2}$. As in [5], we introduce $J_{\boldsymbol{\Gamma}(\mathbf{x}, \mathbf{y})}$ as the Jacobian matrix of $\Gamma$, namely, $\left(J_{\boldsymbol{\Gamma}}\right)_{i j}=\frac{\partial \Gamma_{i}}{\partial y_{j}}$, so that

$$
\nabla_{\mathbf{y}} P_{1}^{V}=-J_{\Gamma(\mathbf{x}, \mathbf{y})}^{T} \nabla_{\mathbf{x}} P_{0}^{V}
$$

We let $p(\mathbf{x}, t)=P_{0}^{V}(\mathbf{x}, t)$ be the homogenized pressure, and

$$
D(\mathbf{x})=I\left|\omega_{V}\right|-\int_{\omega_{V}(\mathbf{x})} J_{\Gamma(\mathbf{x}, \mathbf{y})}^{T} d \mathbf{y}
$$

However, since $a$ is constant, we have constant effective diffusivity $D(\mathbf{x}) \equiv D_{e}$, and so have

$$
\nabla_{\mathbf{x}}^{2} p(\mathbf{x}, t)=\frac{4 \pi \beta}{D_{e}} \frac{p(\mathbf{x}, t) b(\mathbf{x}, t)^{2}}{1+\frac{\beta}{\nu} b(\mathbf{x}, t)\left(1-\frac{b(\mathbf{x}, t)}{a}\right)},
$$

which is coupled to the equation

$$
\frac{\partial b(\mathbf{x}, t)}{\partial t}=-\gamma \frac{p(\mathbf{x}, t)}{1+\frac{\beta}{\nu} b(\mathbf{x}, t)\left(1-\frac{b(\mathbf{x}, t)}{a}\right)} .
$$

We have found these equations by noting that at leading order the pressures in $\omega_{V}$ and $\omega_{S}$ are the same, observing that we can write $P_{1}^{V}$ in terms of $P_{2}^{V}$, and integrating the equation for $P_{2}^{V}$ to relate the homogenized pressure $p$ to the movement of the boundary $b$. The initial condition is $b(\mathbf{x}, 0)=a$, and boundary conditions are $p(1, t)=p_{a}$ and that $p$ is bounded at the origin.

Using the scalings

$$
p=p_{a} \bar{p}, \quad b=a \bar{b}, \quad t=\frac{a}{\gamma p_{a}} \bar{t},
$$

we find, upon dropping the overbar notation and letting $R \in[0,1]$ be the dimensionless macroscale variable, that

$$
\frac{1}{R^{2}} \frac{\partial}{\partial R}\left(R^{2} \frac{\partial p}{\partial R}\right)=\kappa \frac{p b^{2}}{1+\lambda b(1-b)}, \quad 0 \leq R \leq 1, \quad t \geq 0,
$$

Copyright (c) by SIAM. Unauthorized reproduction of this article is prohibited. 


$$
\begin{gathered}
\frac{\partial b}{\partial t}=-\frac{p}{1+\lambda b(1-b)}, \quad 0 \leq R \leq 1, \quad t \geq 0, \\
p(1, t)=1, \quad p \quad \text { bounded as } R \rightarrow 0, \quad b(R, 0)=1,
\end{gathered}
$$

where $\kappa=4 \pi \beta a^{2} / D_{e}, \lambda=a \beta / \nu$. We can also replace (60) with

$$
\frac{1}{R^{2}} \frac{\partial}{\partial R}\left(R^{2} \frac{\partial p}{\partial R}\right)=-\kappa \frac{\partial}{\partial t}\left(\frac{b^{3}}{3}\right), \quad 0 \leq R \leq 1, \quad t \geq 0,
$$

which is global conservation of mass. This represents gas moving into the particle to replace the reacting solid.

4.2. The $\boldsymbol{D}_{\boldsymbol{S}} / \boldsymbol{D}_{\boldsymbol{V}}=\boldsymbol{\delta} \boldsymbol{\nu}$ limit. Returning to system (32)-(36), but with $n=1$, we find that the $\mathcal{O}(1)$ equations give that both $P_{0}^{V}$ and $P_{0}^{S}$ are independent of $\mathbf{y}$, and that $P_{0}^{V}=P_{0}^{S}$. At $\mathcal{O}(\delta)$, we find that $P_{1}^{S}$ satisfies Laplace's equation instead of (42), and that the reaction rate in (44) comes in at lower order, so that the right-hand side is given by $-\frac{\beta}{\nu} P_{0}^{S}$. Equation (41) and the boundary conditions in (43) remain unchanged. At $\mathcal{O}\left(\delta^{2}\right)$, the interfacial pressure flux from $\omega_{S}$ into $\omega_{V}$ is of a lower order, so that the right-hand side of (50) becomes $\nu \nabla_{\mathbf{y}} P_{1}^{S}+\nu \nabla_{\mathbf{x}} P_{0}^{S}$. Integrating (49) (which remains unchanged) over $\omega_{V}$ and using methods analogous to those already used, we can relate the homogeneous pressure $p=P_{0}^{V}=P_{0}^{S}$ to the interface $b$, to obtain the same system (61)-(63), but with $\lambda=0$. Note that the same model arises if a fractional power of $n \in(0,2)$ is instead taken, using an asymptotic expansion in the appropriate fractional powers of $\delta$.

4.3. The $D_{S} / D_{V}=\nu$ limit. If we consider the case where the diffusivities in the void space $\omega_{V}$ and the porous solid $\omega_{S}$ are the same order of magnitude, so $D_{S} / D_{V}=\nu$, then examining the system (32)-(36) with $n=0$ we find that the pressures and fluxes come in at the same order in each region. The lowest order pressures, $P_{0}^{V}$ and $P_{0}^{S}$, are equal and independent of $\mathbf{y}$. We integrate the $\mathcal{O}\left(\delta^{2}\right)$ field equations, using periodicity of $P^{V}$ along $\partial \omega_{o}$ and that the macroscale domain of interest is also spherical, so that $\nabla_{\mathbf{x}}$ is parallel to $\mathbf{y}$. This leads to the homogenized equations

$$
\nabla \cdot\left(\left(D_{e}^{V}+\nu D_{e}^{S}\right) \nabla p\right)=4 \pi \beta b^{2} p, \quad \frac{\partial b}{\partial t}=-\gamma p,
$$

where

$$
D_{e}^{V}(\mathbf{x})=I\left|\omega_{V}\right|-\int_{\omega_{V}(\mathbf{x})} J_{V \boldsymbol{\Gamma}_{V}(\mathbf{x}, \mathbf{y})}^{T} d \mathbf{y}, \quad D_{e}^{S}(\mathbf{x}, t)=I\left|\omega_{S}\right|-\int_{\omega_{S}(\mathbf{x}, t)} J_{S}^{T} \Gamma_{S}(\mathbf{x}, \mathbf{y}) d \mathbf{y},
$$

and $J_{V}$ and $J_{S}$ are analogous to the Jacobian used in (55), that is, $\left(J_{\Gamma_{V}}\right)_{i j}=\frac{\partial \Gamma_{V, i}}{\partial y_{j}}$ and $\left(J_{\boldsymbol{\Gamma}_{S}}\right)_{i j}=\frac{\partial \Gamma_{S, i}}{\partial y_{j}}$, where $\boldsymbol{\Gamma}_{V}$ and $\boldsymbol{\Gamma}_{S}$ are defined in $\omega_{V}$ and $\omega_{S}$, respectively. Using the same scales as in (59), along with $D_{e}^{V}=\overline{D_{e}^{V}} D_{e}$ and $D_{e}^{S}=\overline{D_{e}^{S}} D_{e}$, we obtain the same system as (61)-(63), except with a spatially varying diffusivity $\overline{D_{e}^{V}}+\nu \overline{D_{e}^{S}}$, which is dependent upon the microscale granular behavior, along with $\lambda=0$.

4.4. Other limits. When $n>2$ then we find that $p$ satisfies Laplace's equation and $b$ is spatially independent, so physically it is too hard for the reactant gas to diffuse through $\omega_{S}$ at the granular level to the interface, and so the reaction does not 
take place. In the case $n<0$ we find that $p$ is spatially independent and $b_{t}=-p$, so that the particle converts linearly in time in a spatially homogeneous manner. Although this limit is physically rare, it could occur if a low-porosity binder is used to glue grains together.

4.5. Summary. We can combine each model discussed into a composite model, taking the form

$$
\begin{gathered}
\nabla \cdot\left(\left(\overline{D_{e}^{V}}+\frac{D_{S}}{D_{V}} \overline{D_{e}^{S}}\right) \nabla p\right)=-\kappa \frac{\partial}{\partial t}\left(\frac{b^{3}}{3}\right), \\
\frac{\partial b}{\partial t}=-\frac{p}{1+a \beta \frac{D_{V}}{D_{S}} \delta^{2} b(1-b)},
\end{gathered}
$$

in the domain $0 \leq R \leq 1, t \geq 0$, where $\kappa=4 \pi \beta a^{2} / D_{e}$. As $\delta$ is the ratio of granular to particle lengthscales, we obtain a homogenized model by taking the limit $\delta \rightarrow 0$, with $\kappa, a$, and $\beta$ all $\mathcal{O}(1)$ parameters. If we assume that $D_{S} / D_{V}=\mathcal{O}\left(\delta^{2}\right)$, then we will obtain (60) and (61), since $\overline{D_{e}^{V}}=1$ for this limit. If instead we take $D_{S} / D_{V}=\mathcal{O}(1)$, then (67) becomes $b_{t}=-p$, but we obtain the granular diffusion terms in the first equation. Taking $D_{S} / D_{V}=\mathcal{O}\left(\delta^{n}\right)$ for $n \in(0,2)$ reduces the complexity in both (66) and (67). Since $\lambda=a \beta / \nu$, the $n \in(0,2)$ model can be obtained by taking the $n=2$ model in the limit $\nu \rightarrow \infty$ and the $n=0$ model in the limit $\nu \rightarrow 0$. The $n \in(0,2)$ model is thus a subset of both the $n=0$ and $n=2$ models, which are the two distinguished limits of the homogenization problem. Similarly, taking the $n=2$ model in the limit $\nu \rightarrow 0$ results in the $n>2$ model, and taking the $n=0$ model in the limit $\nu \rightarrow \infty$ results in the $n<0$ model. Since the $n=2$ model is the most physically relevant, along with sublimit $\lambda=0$ corresponding to $n \in(0,2)$, we explore it in the next section.

5. Model behavior. We are interested in the model consisting of (61) and (63) for the spherically symmetric functions $p(R, t)$ and $b(R, t)$, with boundary conditions and initial condition in (62). Upon finding $p$ and $b$, we can use these quantities to determine the concentration of solid $B\left(\mathrm{~mol} / \mathrm{m}^{3}\right)$ and the extent of the reaction. Letting $C(R, t)$ be the dimensionless molar concentration of solid $B$, we have $C(R, t)=$ $b(R, t)^{3}$. The dimensionless extent of the reaction, or degree of conversion, $X(t)$, is the total molar fraction converted, namely,

$$
X(t)=3 \int_{R=0}^{1}\left(1-b^{3}\right) R^{2} d R .
$$

This is a commonly used metric $[18,20,21]$ to summarize the progress of reactions, and we will use it throughout our model analysis. Note that we can utilize (61) and (63) through

$$
\frac{d X}{d t}=-9 \int_{R=0}^{1} \frac{\partial}{\partial t}\left(\frac{b^{3}}{3}\right) R^{2} d R=\frac{9}{\kappa} \int_{R=0}^{1} \frac{\partial}{\partial R}\left(R^{2} \frac{\partial p}{\partial R}\right) d R=\frac{9}{\kappa} \frac{\partial p(1, t)}{\partial R} .
$$

Thus we can find the extent of the reaction by either solving for $b$ or finding $p_{R}$ at the outside of the particle and using the initial condition $X(0)=0$. We analyze the model behavior by first showing some numerical simulations in section 5.1 and then finding asymptotic solutions in the limit of small and large $\kappa$ in section 5.2. 

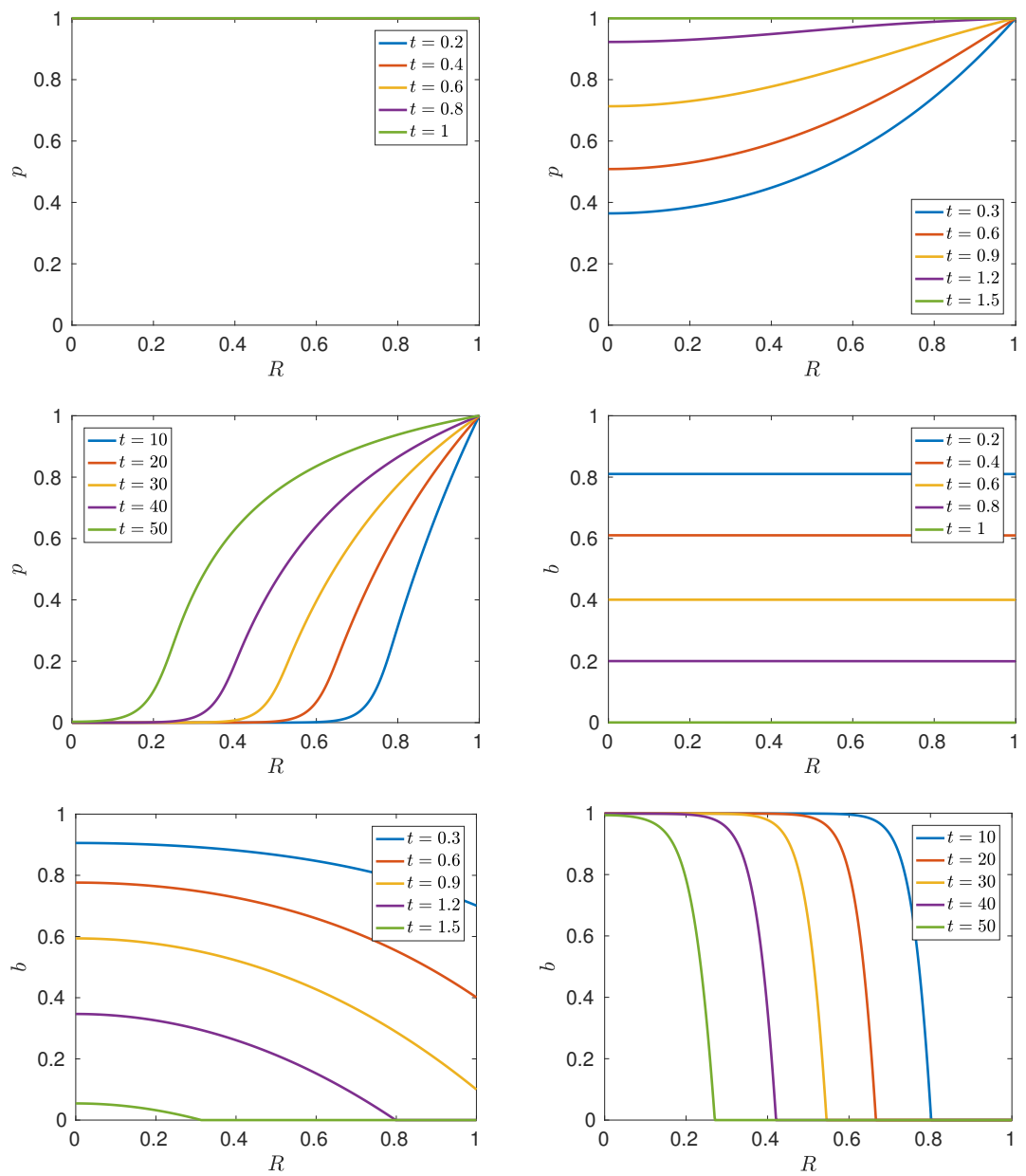

FIG. 3. Numerical simulations of (61)-(63), with $\lambda=0$, for $p$ (top) and $b$ (bottom). From left to right, $\kappa=0.01, \kappa=10$, and $\kappa=1000$. Note how the timescale is different for each choice of $\kappa$. (Color available online.)

5.1. Numerical simulations. We perform numerical simulations using a first order finite difference scheme. In Figure 3, we plot $p$ and $b$ for $\kappa=0.01, \kappa=10$, and $\kappa=1000$, with $\lambda=0$ in all cases. We note that for small $\kappa$ the system is almost spatially homogeneous, with constant pressure $p=1$ and linearly decreasing $b$, but for large $\kappa$ we have an inward-moving wave for $b$. In Figure 4 we plot $X(t)$ for different $\kappa$, again with $\lambda=0$, and also varying $\lambda$ with fixed $\kappa=10$. The larger $\kappa$ is, the longer the reaction takes, but for small $\kappa$, the parameter value has little influence on $X(t)$. Similarly, larger $\lambda$ increases the reaction time, but $X(t)$ is insensitive to changes in $\lambda$ for small values of the parameter. More details about the dependency of $X(t)$ on $\kappa$ and $\lambda$ are given in section 5.2. In Figure 5, we show numerical simulations for $p$ and $b$ with different values of $\kappa$ and $\lambda$. We find that including nonzero $\lambda$ in the model allows for a richer variety of solutions than when $\kappa$ is the only free parameter.

5.2. Asymptotics. We now solve the system (61)-(63) analytically using asymptotic methods, exploiting the size of $\kappa$. We first examine the limit where $\kappa$ is a small 

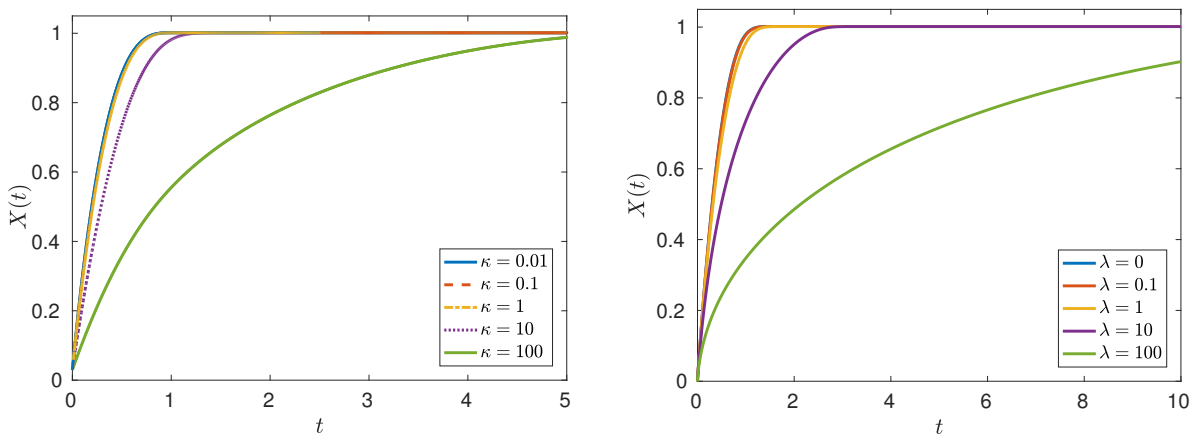

FIG. 4. Extent of reaction against time. In the left plot, $\kappa$ is varied, with $\lambda=0$ fixed. In the right plot, $\lambda$ is varied, with $\kappa=10$ fixed. (Color available online.)
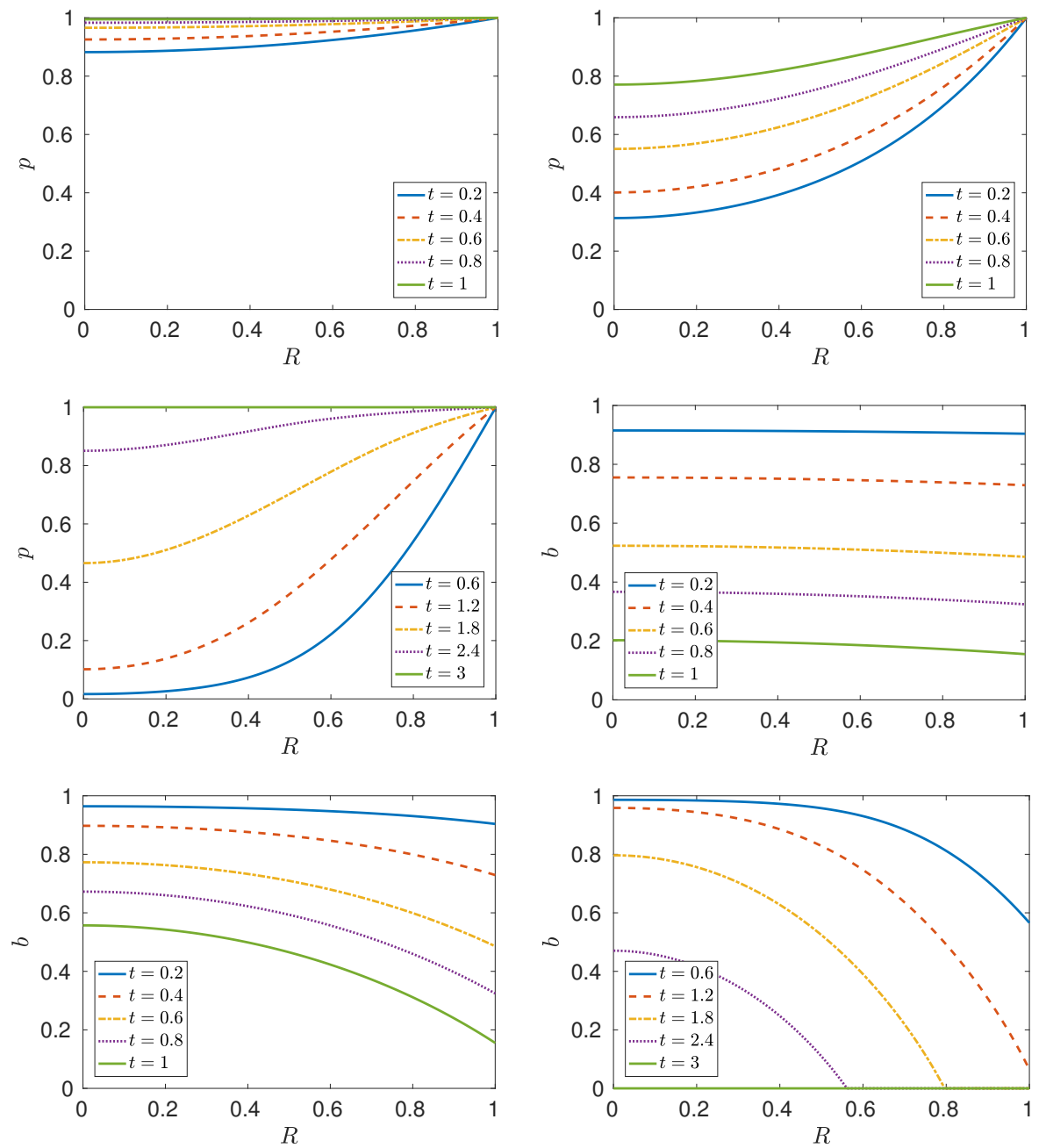

FIG. 5. Numerical simulations of (61)-(63), for $p$ (top) and $b$ (bottom). From left to right parameter values used are $\kappa=1$ and $\lambda=1, \kappa=10$ and $\lambda=1$, and $\kappa=100$ and $\lambda=20$. Note how the timescale is different in the right column. (Color available online.)

Copyright (c) by SIAM. Unauthorized reproduction of this article is prohibited. 
parameter, and next we consider the limit where $\kappa$ is a large parameter. In both cases, we treat $\lambda$ as an $\mathcal{O}(1)$ parameter. In order to make the system easier to solve, we make the substitution $p(R, t)=W(R, t) / R$, which transforms (61)-(63) to (70)-(72) below, which are valid for $0 \leq R \leq 1$ and $t \geq 0$.

$$
\begin{gathered}
\frac{\partial^{2} W}{\partial R^{2}}=\kappa W b^{2}, \\
\frac{\partial b}{\partial t}=-\frac{W}{R} \frac{1}{1+\lambda b(1-b)}, \\
W(1, t)=1, \quad W=\mathcal{O}(R) \quad \text { as } R \rightarrow 0, \quad b(R, 0)=1 .
\end{gathered}
$$

Note that for small $\kappa$, we find it easier to write (70) in the form of (60) rather than in conservation of mass form (63).

5.2.1. Small $\kappa$ limit. We consider the limit $0 \leq \kappa \ll 1$, with $\lambda=\mathcal{O}(1)$, which corresponds physically to a parameter regime where diffusion is fast compared to the kinetic rate, so the particle conversion is rate-limited by the chemical reaction. For this limit, it is helpful to replace (71) with the time-integrated version

$$
b+\frac{\lambda}{2} b^{2}-\frac{\lambda}{3} b^{3}-\left(1+\frac{\lambda}{6}\right)=-\frac{1}{R} \int_{\tau=0}^{t} W(R, \tau) d \tau,
$$

where we integrate over the dummy variable $\tau$. Treating $\kappa$ as a small parameter, we expand $W$ and $b$ as $W=W_{0}+\kappa W_{1}+\kappa^{2} W_{2}+\mathcal{O}\left(\kappa^{3}\right), b=b_{0}+\kappa b_{1}+\kappa^{2} b_{2}+\mathcal{O}\left(\kappa^{3}\right)$. We give solutions here, with the system of equations arising at each order given in Appendix A. We find that

$$
\begin{aligned}
& W(R, t)=1+\frac{\kappa}{6}\left(R^{3}-R\right) f_{0}(t ; \lambda)^{2}+\frac{\kappa^{2}}{6}\left(\frac{R^{5}}{20}-\frac{R^{3}}{6}+\frac{7 R}{60}\right) f_{0}(t)\left(f_{0}(t)^{3}-2 f_{1}(t)\right) \mathcal{O}\left(\kappa^{3}\right), \\
& \quad \begin{aligned}
b(R, t)= & f_{0}(t ; \lambda)+\frac{\kappa}{6}\left(1-R^{2}\right) f_{1}(t ; \lambda) \\
& -\kappa^{2}\left(\frac{1}{6}\left(\frac{R^{4}}{20}-\frac{R^{2}}{6}+\frac{7}{60}\right) f_{21}(t)+\frac{\lambda}{36}\left(R^{2}-1\right)^{2} f_{22}(t)\right)+\mathcal{O}\left(\kappa^{3}\right),
\end{aligned}
\end{aligned}
$$

where the function of time $f_{0}(t ; \lambda)$ is given by the real root of the cubic equation

$$
f_{0}^{3}-\frac{3}{2} f_{0}^{2}-\frac{3}{\lambda} f_{0}+\left(\frac{3}{\lambda}(1-t)+\frac{1}{2}\right)=0,
$$

while $f_{1}(t ; \lambda), f_{21}(t ; \lambda)$, and $f_{22}(t ; \lambda)$ are given by

$$
\begin{aligned}
f_{1}(t ; \lambda) & :=\frac{\int_{\tau=0}^{t} f_{0}(\tau)^{2} d \tau}{1+\lambda f_{0}(t)-\lambda f_{0}(t)^{2}}, \quad f_{21}:=\frac{\int_{\tau=0}^{t} f_{0}(\tau)\left(f_{0}(\tau)^{3}-2 f_{1}(\tau)\right) d \tau}{1+\lambda f_{0}(t)\left(1-f_{0}(t)\right)}, \\
f_{22} & :=\frac{f_{1}(t)^{2}\left(\frac{1}{2}-f_{0}(t)\right)}{1+\lambda f_{0}(t)\left(1-f_{0}(t)\right)} .
\end{aligned}
$$

Using (75) in (68), we find

$$
X(t)=1-f_{0}(t)^{3}-\frac{\kappa}{5} f_{0}^{2} f_{1}+\frac{2 \kappa^{2}}{105}\left(f_{0}^{2} f_{21}+\lambda f_{0}^{2} f_{22}-f_{0} f_{1}^{2}\right)+\mathcal{O}\left(\kappa^{3}\right) .
$$




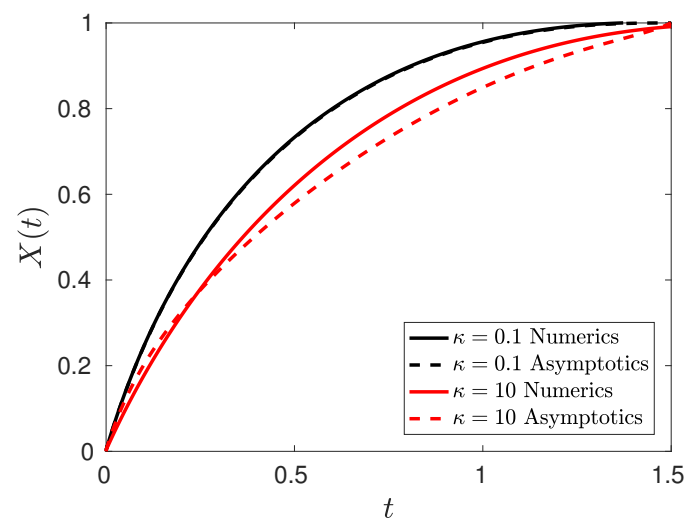

FIG. 6. Comparison of numerics (solid) and asymptotics (dashed) for the extent of the reaction $X(t)$, with $\lambda=3$. Even for $\kappa=10$, the small $\kappa$ asymptotics give a decent agreement with numerics. (Color available online.)

The asymptotic solution (78) is compared to numerics in Figure 6, for two values of $\kappa$, but the same value of $\lambda$. The time $t=t^{*}$ for the reaction to be completed is given implicitly, to leading order, by $f_{0}\left(t^{*}\right)=0$, which from (76) we find $t^{*}=1+\lambda / 6$.

Notice that when $\lambda=0$ we have

$$
f_{0}(t)=1-t, \quad f_{1}(t)=\frac{1}{3}\left(1-(1-t)^{3}\right), \quad f_{21}(t)=\frac{1}{3}\left((1-t)^{2}-(1-t)^{5}\right),
$$

and so we have the solutions

$W(R, t)=R-\frac{\kappa}{6}(1-t)^{2}\left(R-R^{3}\right)+\frac{\kappa^{2}}{9}\left(\frac{5}{2}(1-t)^{4}-(1-t)\right)\left(\frac{R-R^{3}}{6}-\frac{R-R^{5}}{20}\right)+\mathcal{O}\left(\kappa^{3}\right)$,

$$
\begin{aligned}
b(R, t)= & (1-t)+\frac{\kappa}{18}\left(1-\left(1-t^{3}\right)\right)\left(1-R^{2}\right) \\
& +\frac{\kappa^{2}}{18}\left((1-t)^{5}-(1-t)^{2}\right)\left(\frac{1-R^{2}}{6}-\frac{1-R^{4}}{20}\right)+\mathcal{O}\left(\kappa^{3}\right),
\end{aligned}
$$

$$
X(t)=1-(1-t)^{3}+\frac{\kappa}{15}\left((1-t)^{5}-(1-t)^{2}\right)-\frac{2 \kappa^{2}}{945}\left(4(1-t)^{7}-5(1-t)^{4}+(1-t)\right)+\mathcal{O}\left(\kappa^{3}\right) .
$$

Although the results have used the limit $\kappa \rightarrow 0$, in practice for $\kappa<15$ the series (82) appears to be a good approximation, but for $\kappa>15$ the series (82) ceases to be asymptotic, and it is no longer a helpful approximation. The series given by (80), (81), and (82) are only valid for $t \in[0,1]$.

5.2.2. Large $\kappa$ limit. We now consider the case where $\kappa$ is a large parameter, with $\lambda$ still $\mathcal{O}(1)$, for the system (61)-(63). This corresponds to the particle conversion being rate-limited by diffusion, which is slow compared to the kinetic rate. We find that the reaction occurs on an $\mathcal{O}(\kappa)$ timescale, and so we introduce the $\mathcal{O}(1)$ variable $\tau=t / \kappa$. This transforms $(61)$ to

$$
\frac{\partial b}{\partial \tau}= \begin{cases}\kappa \frac{W}{R} \frac{1}{1+\lambda b(1-b)}, & b \geq 0, \quad 0 \leq R \leq 1, \quad \tau \geq 0, \\ 0, & b<0,\end{cases}
$$

Copyright $@$ by SIAM. Unauthorized reproduction of this article is prohibited. 
where we have included the case $b<0$ to ensure that $b$ does not become negative, which would be unphysical. Expanding in $\kappa^{-1}$ like $W=W_{0}+\frac{1}{\kappa} W_{1}+\mathcal{O}\left(\frac{1}{\kappa^{2}}\right), b=$ $b_{0}+\frac{1}{\kappa} b_{1}+\mathcal{O}\left(\frac{1}{\kappa^{2}}\right)$, we obtain

$$
\begin{gathered}
b_{0}(R, \tau)= \begin{cases}0, & R \geq s(\tau), \\
1, & R<s(\tau),\end{cases} \\
W_{0}(R, \tau)= \begin{cases}\omega(R, \tau), & R \geq s(\tau), \\
0, & R<s(\tau),\end{cases}
\end{gathered}
$$

for some unknown function of time $s(\tau)$ and linear function $\omega(R, \tau)=1+Y(\tau)(R-1)$.

To determine $s(\tau)$ and $Y(\tau)$, we note that there must be a boundary layer of thickness $\mathcal{O}\left(\frac{1}{\sqrt{\kappa}}\right)$ in $R$ in order to balance the derivative term in (83) with the $\mathcal{O}(\kappa)$ term on the right-hand side. To find the behavior in this boundary layer, we introduce the scalings

$$
\eta=\sqrt{\kappa}(s(\tau)-R), \quad W(R, t)=\sqrt{\kappa} v(\eta, \tau), \quad b(R, t)=h(\eta, \tau)
$$

and use the conservation form of the equation

$$
\frac{\partial^{2} W}{\partial R^{2}}=-\frac{1}{3} R \frac{\partial}{\partial \tau}\left(b^{3}\right)
$$

instead of (61). With these scalings, we obtain the system for $v, h$, and $s$, for $\tau \geq 0$ and $\eta \geq 0$,

$$
\begin{gathered}
\frac{\partial^{2} v}{\partial \eta^{2}}=-s \frac{d s}{d \tau} h^{2} \frac{\partial h}{\partial \eta}+\mathcal{O}\left(\frac{1}{\sqrt{\kappa}}\right), \quad \frac{d s}{d \tau} \frac{\partial h}{\partial \eta}=-\frac{v}{s(1+\lambda h(1-h))}+\mathcal{O}\left(\frac{1}{\sqrt{\kappa}}\right), \\
h(0, \tau)=0, \quad h \rightarrow 1 \quad \text { as } \eta \rightarrow \infty, \quad v \rightarrow 0 \quad \text { as } \eta \rightarrow \infty,
\end{gathered}
$$

along with two equations arising from suitable matching of $v$ and $W$ and their derivatives at $\eta=0$ (that is, $R=s(\tau)$ ).

Taking the leading order terms from (88) (which can be found by expanding $v=v_{0}+\mathcal{O}\left(\frac{1}{\sqrt{\kappa}}\right), h=h_{0}+\mathcal{O}\left(\frac{1}{\sqrt{\kappa}}\right)$, but we suppress the 0 subscript), then a first integral of the first equation in (88) can be taken to give

$$
\frac{\partial v}{\partial \eta}=s \frac{d s}{d \tau}\left(\frac{1}{3}-\frac{h^{3}}{3}\right) .
$$

Upon integrating, the arbitrary function of time was determined by the last two conditions in (89), assuming that $v$ is suitably well behaved so that $v_{\eta} \rightarrow 0$ as $\eta \rightarrow \infty$. Since we also have from the second equation in (88) that

$$
v=-s \frac{d s}{d \tau} \frac{\partial h}{\partial \eta}(1+\lambda h(1-h))
$$

we can substitute (91) into (90) to obtain the second order ODE in $\eta$ for $h(\eta, \tau)=$ $F(\eta)$, which reads

$$
\frac{d}{d \eta}\left(\frac{d F}{d \eta}(1+\lambda F(1-F))\right)=\frac{F^{3}}{3}-\frac{1}{3}, \quad F(0)=0, \quad F(\infty)=1 .
$$


Note that we can find $F(\eta ; \lambda)$ numerically by writing the ODE as a first order system for $F$ and $G=F^{\prime}$, where ' denotes differentiation with respect to $\eta$. We then vary the initial value of $G$ until $F(N)$ is sufficiently close to 1 , for large $N$ (we shall take $N=$ 20 ). This shooting method is highly sensitive to the initial conditions, since the ODE is unstable at $\eta=\infty, F=1$. This can be seen by letting $F=1-\theta$, with $0<\theta \ll 1$, so that upon linearizing (92) we find $\theta^{\prime \prime} \sim 3 \theta$, and hence $\theta \sim A_{0} e^{\sqrt{3} \eta}+B_{0} e^{-\sqrt{3} \eta}$. In Figure 7, we show a plot of $F$ for varying $\lambda$.

In the case where $\lambda=0$, a first integral of (92) can be obtained, and this takes the form

$$
\frac{d F}{d \eta}=\sqrt{\frac{F^{4}}{6}-\frac{2 F}{3}+\frac{1}{2}}, \quad F(0)=0,
$$

where the positive square root is taken because $F$ is an increasing positive function. Since $F(5 ; 0) \sim 0.99$, then when $\lambda=0$ the boundary layer is of width $\eta \sim 5$, which in radial coordinates is $R \sim 5 / \sqrt{\kappa}$.

If the parameter $\lambda$ is increased such that $\lambda \gg 1$, then at leading order (92) becomes

$$
\frac{d^{2}}{d \eta^{2}}\left(\frac{F^{2}}{2}-\frac{F^{3}}{3}\right)=0
$$

Integrating (94) twice gives $\frac{F^{2}}{2}-\frac{F^{3}}{3}=A_{1} \eta+B_{1}$, and so $F$ cannot satisfy both boundary conditions $F(0)=0$ and $F(\infty)=1$.

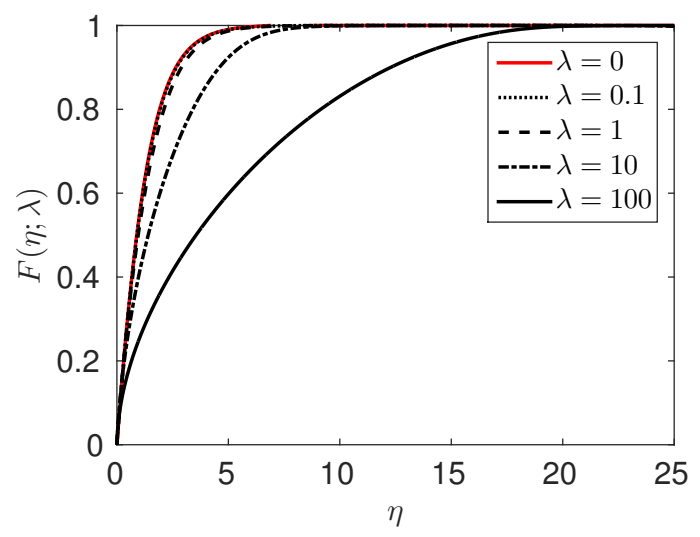

FIG. 7. Numerical plots of $F(\eta ; \lambda)$, for varying $\lambda$. (Color available online.)

Returning to study $h$ and $v$, using $h(0, \tau)=F(0)=0$ with (90) and (91) gives

$$
v=-s \frac{d s}{d \tau} \frac{d F}{d \eta}, \quad \frac{\partial v}{\partial \eta}=\frac{s}{3} \frac{d s}{d \tau} \text { at } \eta=0
$$

In order to match $s$ from the right-hand side, we let $\omega(R, \tau)=1+Y(\tau)(R-1)$, with

$$
\omega(R, \tau)=\frac{v(\eta, \tau)}{\sqrt{\kappa}}, \quad \frac{\partial \omega(R, \tau)}{\partial R}=\frac{\partial v(\eta, \tau)}{\partial \eta} .
$$

Thus, by continuity of $v$ and $\omega$, and their derivatives, it is evident that

$$
1+Y(\tau)(s-1)=-\frac{1}{\sqrt{\kappa}} s \frac{d s}{d \tau} \frac{d F}{d \eta}(0), \quad \frac{s}{3} \frac{d s}{d \tau}=Y(\tau) .
$$

Copyright $@$ by SIAM. Unauthorized reproduction of this article is prohibited. 
Taking the limit $\kappa \rightarrow \infty$, and assuming that $F^{\prime}(0)=o(\sqrt{\kappa})$, so that $F^{\prime}(0) / \sqrt{\kappa} \rightarrow 0$ as $\kappa \rightarrow \infty$, we have that $1+Y(\tau)(s-1)=0$, and we obtain

$$
\omega(R, t)=\frac{R-s(\tau)}{1-s(\tau)},
$$

along with the ODE for $s$,

$$
\frac{d s}{d \tau}=-\frac{3}{s(s-1)}, \quad s(0)=1 .
$$

The ODE (99) is solved by the implicit cubic

$$
\frac{s(\tau)^{3}}{3}-\frac{s(\tau)^{2}}{2}+\frac{1}{6}=3 \tau,
$$

which in terms of $t$ is

$$
\frac{s(t)^{3}}{3}-\frac{s(t)^{2}}{2}+\frac{1}{6}=\frac{3 t}{\kappa}
$$

This result is encouraging, as it is of the same form as the implicit cubic relationship in (20), taken in the limit $\hat{\beta} \rightarrow \infty$ with $\hat{\beta} / \hat{\gamma}$ finite, and hence the slow diffusion limit of our homogenized model matches the slow diffusion limit of the shrinking core model, up to a timescale. This diffusion-controlled regime is discussed in more depth in section 7 .

The time $t=t^{*}$ for the reaction to be completed is found by setting $s\left(t^{*}\right)=0$ in (101), to give $t^{*}=\frac{\kappa}{18}$, which, interestingly, is independent of $\lambda$. This parameter influences the shape of $b$ in the boundary layer (as seen in Figure 7), but has no influence on the time for the reaction to finish. Numerical simulations support this claim, with a relative variation in $t^{*}$ of $1.03 \%$ between $\lambda=0$ and $\lambda=10$ for $\kappa=1000$. However, a larger variation is found when $\lambda \sim \sqrt{\kappa} \sim 31.6$. We can determine when the assumption that $F^{\prime}(0)=o(\sqrt{\kappa})$ is not suitable, and so (98) no longer holds, since $F^{\prime}(0) / \sqrt{\kappa} \nrightarrow 0$ in the limit $\kappa \rightarrow \infty$. When $\lambda=0$, then $F^{\prime}(0)=\frac{1}{\sqrt{2}}$, which is sufficient. For general $\lambda>0$ we have

$$
F^{\prime \prime}(0)+F^{\prime}(0)^{2} \lambda=-\frac{1}{3},
$$

which means that $F^{\prime \prime}(0)<0$ and that for large $\lambda$ we need either $F^{\prime}(0)=\mathcal{O}\left(\lambda^{-2}\right)$ and $F^{\prime \prime}(0)=\mathcal{O}(1)$, or $F^{\prime}(0)=\mathcal{O}\left(\lambda^{-1}\right)$ and $F^{\prime \prime}(0)=\mathcal{O}\left(\lambda^{-1}\right)$. Thus the analysis can break down for $\lambda=\mathcal{O}\left(\frac{1}{\sqrt{\kappa}}\right)$.

We thus have leading order solutions for $b$ and $W$, where $\eta=(\sqrt{\kappa}(s(t)-R))$, which are given by

$$
\begin{gathered}
b(R, t)= \begin{cases}0, & R \geq s(t), \\
F(\eta), & R \leq s(t),\end{cases} \\
W(R, t)= \begin{cases}\frac{R-s(t)}{1-s(t)}, \\
\frac{3}{\sqrt{\kappa}} \frac{3}{1-s(t)} F^{\prime}(\eta)(1+\lambda F(\eta)(1-F(\eta))), & R \leq s(t) .\end{cases}
\end{gathered}
$$

The extent of reaction $X(t)$ is found from (68) by noting $b \rightarrow \mathcal{H}(s(t)-R)$ as $\kappa \rightarrow \infty$, to give

$$
X(t) \sim 1-s(t)^{3} .
$$

Asymptotic results for $s(t)$ and $X(t)$ are compared to numerics in Figure 8. 


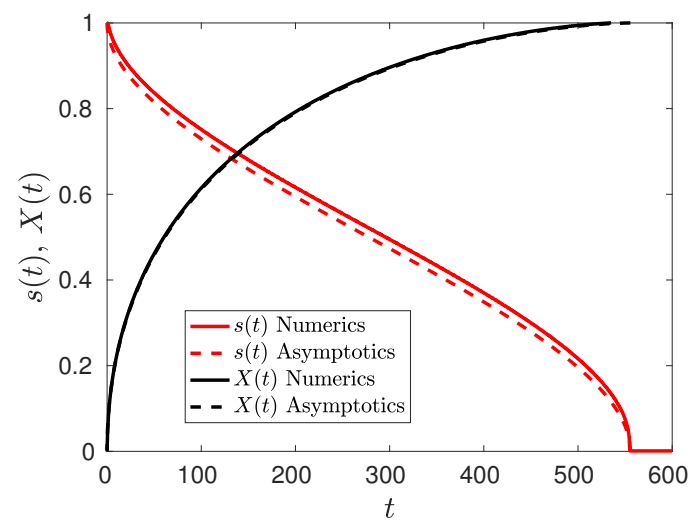

FIG. 8. Comparison of numerics and asymptotics for $s(t)$ (red) and $X(t)$ (black) for $\kappa=10^{4}$ and $\lambda=0$. (Color available online.)

5.2.3. Summary of asymptotics. System (61)-(63) arises from considering the distinguished limit $D_{S} / D_{V}=\delta^{2} \nu$ in section 4 and is the most physically relevant model. This model depends on two parameters, $\kappa$ and $\lambda$, and has been analyzed in the limit $\kappa \rightarrow 0$ and $\kappa \rightarrow \infty$, with $\lambda=\mathcal{O}(1)$ in each case. When $\kappa \rightarrow 0$, we find that the system is spatially homogeneous to leading order. Taking $\lambda=0$ reduces the complexity of the resulting solutions. For large $\kappa$ we find a sharp inward-moving wave, separating a reacted exterior from an unreacted core, as with the canonical shrinking core problem discussed in section 2 , but with a boundary layer ahead of the interface of size $R=\mathcal{O}(1 / \sqrt{\kappa})$. Taking $\lambda=0$ results in a first order ODE to be solved for the shape of $b$ in this boundary layer, rather than a second order ODE when $\lambda>0$.

In each case, we find a leading order prediction of the overall time for reaction, $t=t^{*}$. In the limit $\kappa \rightarrow 0$, we have $t^{*} \sim 1+\lambda / 6$, whereas in the limit $\kappa \rightarrow \infty$, we have $t^{*} \sim \kappa / 18$. Note that these can be combined to give an overall prediction for $t^{*}$, covering the whole range of $\kappa$, which is $t^{*}=1+\lambda / 6+\kappa / 18$. This combination matches well with numerics, as seen in Figure 9.

6. Influence of product gas. In sections $2-5$, we considered a simplified physical setup where the partial pressure of the product gas $F$ has no influence on the reaction. However, we can easily extend the homogenization in section 3 to include the effect of binary gas diffusion and determine the influence on the overall reaction rate, with $P$ and $Q$ referring to the partial pressures of gases $A$ and $F$, respectively. This is important, since for some reactions the counterdiffusion of the product gas can be the rate-limiting step, slowing down the transport of the reactant gas to the reaction fronts. At the microscale reaction interface, one mole of the reactant gas $A$ forms $\sigma_{2}$ moles of the product gas $F$, according to the reaction (1).

Although several models for binary gas diffusion are commonly used $[6,13,16]$, we utilize a simple linear Fickian model here, since it allows a straightforward extension of our findings. Fu et al. [12] have shown that both the dusty gas model and the Stefan-Maxwell model give better representations of experimental behavior in porous electrodes than the multicomponent Fickian diffusion model does. However, it is not yet clear how to use these models to build upon the rest of the analysis in our paper. Instead, we take the simplest approach for modeling the influence of product gas, but are aware of the limitations for some applications. 


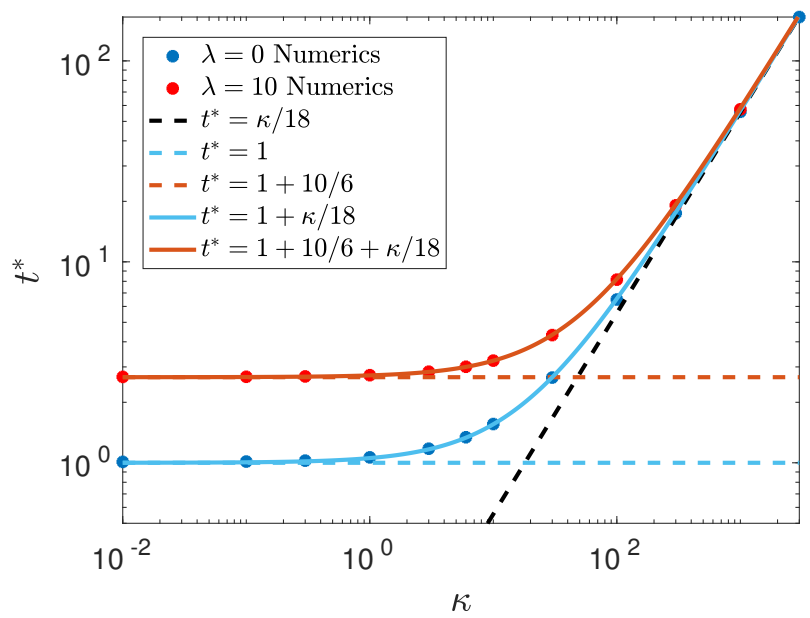

FIG. 9. Comparison of asymptotics and numerics for time for complete reaction $t^{*}$ for varying $\kappa$, with $\lambda=0$ in blue and $\lambda=10$ in red. The plot is on a logarithmic scale. Note how the combined asymptotic result $t^{*}=1+\lambda / 6+\kappa / 18$ matches well with the numerics for both values of $\lambda$. (Color available online.)

We assume that dimensionless pressures $P^{V}$ and $Q^{V}$ exist in $\omega_{V}$, while $P^{S}$ and $Q^{S}$ exist in $\omega_{S}$. In both $\omega_{V}$ and $\omega_{S}$, the diffusion equations will be of the form

$$
\alpha \frac{\partial}{\partial t}\left(\begin{array}{l}
P \\
Q
\end{array}\right)=\nabla \cdot\left(\left(\begin{array}{cc}
1 & d_{1} \\
d_{2} & d_{3}
\end{array}\right) \nabla\left(\begin{array}{l}
P \\
Q
\end{array}\right)\right)=\left(\begin{array}{cc}
1 & d_{1} \\
d_{2} & d_{3}
\end{array}\right) \nabla^{2}\left(\begin{array}{l}
P \\
Q
\end{array}\right)
$$

for some parameter $\alpha$. We also assume that the diffusivities $d_{1}, d_{2}$, and $d_{3}$ are all $\mathcal{O}(1)$ and that the determinant of the diffusion tensor, $d_{3}-d_{1} d_{2}$, is $\mathcal{O}(1)$ in size and positive, to ensure positive definiteness of the matrix. If this were not true, the problem would be degenerate; for example, in the specific singular case $d_{1}=d_{2}=d_{3}=0$ the pressure of product gas would remain constant, corresponding to the single gas case considered earlier.

Upon premultiplying (106) by the matrix inverse (provided the determinant is nonzero), we obtain the rotated equations

$$
\frac{\alpha}{d_{3}-d_{1} d_{2}}\left(\begin{array}{cc}
d_{3} & -d_{1} \\
-d_{2} & 1
\end{array}\right) \frac{\partial}{\partial t}\left(\begin{array}{l}
P \\
Q
\end{array}\right)=\nabla^{2}\left(\begin{array}{l}
P \\
Q
\end{array}\right) .
$$

Hence, for the top-left entry in the diffusion tensor in $\omega_{V}$ being the dimensional diffusion scale $D_{11}:=D_{V}$, then, letting $D_{S} / D_{V}=\nu \delta^{n}$, we obtain the system

$$
\begin{gathered}
\frac{\epsilon}{d_{3}-d_{1} d_{2}}\left(\begin{array}{cc}
d_{3} & -d_{1} \\
-d_{2} & 1
\end{array}\right) \frac{\partial}{\partial t}\left(\begin{array}{l}
P^{V} \\
Q^{V}
\end{array}\right)=\nabla^{2}\left(\begin{array}{l}
P^{V} \\
Q^{V}
\end{array}\right), \quad \mathbf{y} \in \omega_{V}, \\
\frac{\epsilon / \delta^{n} \nu}{d_{3}-d_{1} d_{2}}\left(\begin{array}{cc}
d_{3} & -d_{1} \\
-d_{2} & 1
\end{array}\right) \frac{\partial}{\partial t}\left(\begin{array}{l}
P^{S} \\
Q^{S}
\end{array}\right)=\nabla^{2}\left(\begin{array}{c}
P^{S} \\
Q^{S}
\end{array}\right), \quad \mathbf{y} \in \omega_{S}, \\
\left(\begin{array}{l}
P^{V} \\
Q^{V}
\end{array}\right)=\left(\begin{array}{c}
P^{S} \\
Q^{S}
\end{array}\right), \quad \nabla\left(\begin{array}{l}
P^{V} \\
Q^{V}
\end{array}\right)=\nu \delta^{n} \nabla\left(\begin{array}{l}
P^{S} \\
Q^{S}
\end{array}\right) \quad \text { at } r=a,
\end{gathered}
$$

Copyright $\odot$ by SIAM. Unauthorized reproduction of this article is prohibited. 
(111)

$-\delta^{n} \nu\left(\begin{array}{cc}1 & d_{1} \\ d_{2} & d_{3}\end{array}\right) \nabla\left(\begin{array}{c}P^{S} \\ Q^{S}\end{array}\right) \cdot \mathbf{n}+\epsilon\left(\begin{array}{c}P^{S} \\ Q^{S}\end{array}\right) \frac{\partial b}{\partial t}=\delta \beta P^{S}\left(\begin{array}{c}-1 \\ \sigma_{2}\end{array}\right), \quad \frac{\partial b}{\partial t}=-\gamma P^{S} \quad$ at $r=b$.

We use the usual multiple scales definitions (30) and (31) and assume that $\epsilon$ is sufficiently small so as to not enter the analysis. With this assumption, we find that the problems for the reactant pressure $P$ and product pressure $Q$ decouple (see Appendix B). Introducing the parameter

$$
\sigma:=\frac{d_{3}+d_{1} \sigma_{2}}{d_{3}-d_{1} d_{2}}
$$

for stoichiometric coefficient $\sigma_{2}$ given in (1), we have the same system for $P$ as in (32)-(36), but with $\beta$ replaced by $\sigma \beta$. Thus, upon expanding $P^{V}$ and $P^{S}$ in terms of $\delta$, we would obtain the same homogenized equations as before, but with $\sigma \beta$ in place of $\beta$. Note that $\sigma=1$ corresponds to $d_{1}=0$ (since $\sigma_{2}, d_{2} \geq 0$ ). In most practical situations the off-diagonal "cross-diffusion" terms $d_{1}$ and $d_{2}$ are equal. Thus, when there is no diffusive interaction between the product and reactant gas, we have the same models as if there were no reactant gas.

We can include binary gas diffusion into the homogenized models by adapting the composite model of (66) and (67) to

$$
\begin{gathered}
\nabla \cdot\left(\left(\overline{D_{e}^{V}}+\frac{D_{S}}{D_{V}} \overline{D_{e}^{S}}\right) \nabla p\right)=-\kappa \sigma \frac{\partial}{\partial t}\left(\frac{b^{3}}{3}\right), \quad 0 \leq R \leq 1, \quad t \geq 0, \\
\frac{\partial b}{\partial t}=-\frac{p}{1+\sigma a \beta \frac{D_{V}}{D_{S}} \delta^{2} b(1-b)}, \quad 0 \leq R \leq 1, \quad t \geq 0 .
\end{gathered}
$$

Hence, all of the asymptotic results from section 5.2 still hold, but with the alterations $\kappa \rightarrow \sigma \kappa$ and $\lambda \rightarrow \sigma \lambda$, and in particular the overall time for reaction is predicted by

$$
t^{*}=1+\frac{\sigma \lambda}{6}+\frac{\sigma \kappa}{18}
$$

Note that $\sigma \geq 1$ for a positive definite diffusion tensor, so including the reactant gas in the model will always slow down the reaction time. One could consider the singular limit $\sigma \rightarrow \infty$, but we do not examine this here, as it is not interesting for our physical results.

7. Comparison to existing results. We now compare our findings to those already present in the literature. In section 7.1, we compare the shrinking core model of section 2 to existing formulations, noting the simplifications when the limiting step in the process is diffusion or the chemical reaction. In section 7.2 , we comment on the times for complete particle reaction found from asymptotic analysis in section 5.2 of the homogenized model equations given in section 3. We then comment on Sohn's "law of additive reaction times" [27] in section 7.3, and finally we discuss how our homogenized model fits in with other continuum models for gas-solid reactions in section 7.4.

7.1. Comparison with existing shrinking core models. The shrinking core model in section 2 is found in the literature $[15,21,28,33,36]$, where it is often written as a relationship between time, $t$, and the extent of reaction, $X(t)$. This relationship 
is written in the form $t=h(X)$. An ODE can be found for $X(t)$ upon differentiating this relationship with respect to $t$, and rearranging. Doing so, we have

$$
\frac{d X}{d t}=\frac{1}{h^{\prime}(X)} .
$$

The form of $h$ is dependent on the particle geometry and the particular controlling mechanism. In this paper we have considered models for a spherical geometry. A distinction is often made between when the reaction is controlled by diffusion through the product layer and when it is controlled by the chemical reaction at the interface $l(t)$. In each case, the slowest process is the controlling factor. When the reaction is diffusion-controlled, $h$ is given by $h_{d}$, and when the chemical reaction controls the process, $h$ is given by $h_{r}$, where typical functional forms for each are given in $[15,21,28,33,36]$ by

$$
h_{d}=\frac{1}{k_{d}}\left(1-3(1-X)^{2 / 3}+2(1-X)\right), \quad h_{r}=\frac{1}{k_{r}}\left(1-(1-X)^{1 / 3}\right) .
$$

Thus the time for complete reaction $t^{*}$ in each case is given by $t_{d}^{*}=k_{d}^{-1}$ and $t_{r}^{*}=k_{r}^{-1}$, respectively. Gbor and Jia [15] give

$$
k_{d}=\frac{6 \sigma_{1} D_{\mathrm{eff}} C_{A 0}}{\rho R_{p}^{2}}, \quad k_{r}=\frac{\sigma_{1} k_{1} C_{A 0}^{n}}{\rho R_{p}}
$$

where $D_{\text {eff }}\left(\mathrm{m}^{2} / \mathrm{s}\right)$ is the effective diffusion coefficient of gas $A$ through the product layer, $C_{A 0}\left(\mathrm{~mol} / \mathrm{m}^{3}\right)$ is the concentration of gas $A$ outside the particle, $\rho\left(\mathrm{mol} / \mathrm{m}^{3}\right)$ is the molar density of $B$ in the solid (the same as $\left.B_{0}=\rho_{B} / M_{B}\right), n$ is the order of reaction with respect to $A$, and $k_{1}\left(\mathrm{~mol}^{(1-n)} \mathrm{m}^{(3 n-2)} \mathrm{s}^{-1}\right)$ is the chemical reaction rate constant. Similar constants for $k_{r}$ and $k_{d}$ are given in [21]. Szekely, Evans, and Sohn [33] include the equilibrium constant $K_{E}$, concentration of product gas $F$ outside the particle $C_{F 0}\left(\mathrm{~mol} / \mathrm{m}^{3}\right)$, and reaction order $m$ with respect to $F$, to write

$$
k_{d}=\frac{K_{E}}{1+K_{E}}\left(C_{A 0}-\frac{C_{F 0}}{K_{E}}\right)^{+} \frac{6 b D_{\mathrm{eff}}}{\rho R_{p}^{2}}, \quad k_{r}=\frac{\sigma_{1} k_{1}}{\rho R_{p}}\left(C_{A 0}^{n}-\frac{C_{F 0}^{m}}{K_{E}}\right)^{+},
$$

where we have added the ${ }^{+}$notation to indicate that $k_{d}$ and $k_{r}$ cannot be negative. Equation (119) is a more general result than (118), including thermodynamic effects in the reaction model. However, to keep our model somewhat simple, we ignored these effects in our first order reaction model (7).

Note that considering limits of the shrinking core model (20), we obtain the results in (117). Since $l(t)=(1-X)^{1 / 3}$, then in the limit $\hat{\beta} \rightarrow 0$ with constant external dimensionless pressure $p_{a}$, we obtain the dimensionless relationship

$$
l(t)=1-\hat{\gamma} p_{a} t
$$

which rearranges to the second equation in (117), with $k_{r}$ as in (118). This limit corresponds to infinitely fast diffusion, and so the process is reaction controlled. Instead, taking (20) in the limit $\hat{\beta} \rightarrow \infty$, with $\hat{\beta} / \hat{\gamma}$ finite and for constant pressure $p_{a}$, we obtain

$$
\frac{l(t)^{3}}{3}-\frac{l(t)^{2}}{2}+\frac{1}{6}=\frac{\hat{\gamma}}{\hat{\beta}} p_{a} t
$$


which rearranges to the first equation in (117), with $k_{d}$ as in (118). This limit corresponds to the kinetic rate $k \rightarrow 0$, so that the process is diffusion-controlled. Thus, the simple model outlined in section 2 supports the diffusion-limited and reaction-limited simplifications found in the literature.

7.2. Physical results from our asymptotics. Composing the original dimensionless time $\hat{t}=t / \tau_{s}$ (given in (22)) and the timescale $\bar{t}=\left(\gamma p_{a} / a\right) \hat{t}$ (given in (59)) for the model arising from homogenization, we then use the dimensionless time $t^{*}=1+\sigma \lambda / 6+\sigma \kappa / 18$ to obtain the prediction of the dimensional time for complete reaction $t=t_{\mathrm{dim}}^{*}(\mathrm{~s})$, as

$$
t_{\mathrm{dim}}^{*}=\frac{a R_{p}}{\sigma_{1} k p_{a} P_{\mathrm{TOT}}}+\sigma \frac{a^{2} R_{p}^{2} B_{0}\left(k_{m} / \delta\right)}{6 \sigma_{1} k C_{A}} \frac{\delta^{2}}{D_{S}}+\sigma \frac{4 \pi a^{3}}{3 D_{e}} \frac{R_{p}^{2} B_{0}\left(k_{m} / \delta\right)}{6 \sigma_{1} k C_{A} D_{V}} .
$$

The first two terms correspond to the small $\kappa$ limit where diffusion in the void space $\omega_{V}$ is fast compared to the kinetic rate, and the third term corresponds to the large $\kappa$ limit, where diffusion in the void space is the slow, rate-limiting process. The first term represents the chemical reaction at the microscale granular interface, while the second term represents diffusion through the microscale $\omega_{S}$ solid product matrix. We thus have that the total reaction time is the sum of the time under the limit of three effects, which are the kinetic rate, the microscale diffusion through $\omega_{S}$, and the macroscale diffusion through $\omega_{V}$.

Results from existing models only account for the first and third terms. Assuming that $k_{m}=\delta k$, then for small $\delta$ the second term will be negligible. We can relate the time for kinetic control, $t_{1}^{*}$, and pore-diffusion control, $t_{2}^{*}$, to $t_{r}^{*}=k_{r}^{-1}$ and $t_{d}^{*}=k_{d}^{-1}$, respectively, with $k_{r}$ and $k_{d}$ found by Gbor and Jia [15] and given in (118), through

$$
t_{1}^{*}=\sigma \frac{a R B_{0}}{\sigma_{1} k_{1} C_{A}}=\sigma a t_{r}^{*}, \quad t_{2}^{*}=\sigma \frac{4 \pi a^{3}}{3 D_{e}} \frac{D_{\mathrm{eff}}}{D_{V}} t_{d}^{*}
$$

Here, $D_{V}\left(\mathrm{~m}^{2} / \mathrm{s}\right)$ is the diffusivity of gas $A$ in a void space, which is a measurable quantity. $D_{\text {eff }}\left(\mathrm{m}^{2} / \mathrm{s}\right)$ is the effective diffusivity of gas $A$ throughout the porous macroscale particle, which is in theory measurable for each type of particle used in experiments relevant to industrial applications. The parameter $a$ is the radius of the reactant solid spherical grain in the microscale, assuming without loss of generality that the microscale cell is of unit volume. Since we have assumed a cubic packing arrangement, then, letting each cell be a unit cube, we have $a=1 / 2$. The effective diffusivity $D_{e}$ is given by (56) (for $D_{e}=D(\mathbf{x})$ ). For a cubic packing with $a=1 / 2$, Bruna and Chapman [5] have found $D_{e}=0.72$ using COMSOL Multiphysics (see Figure $3 \mathrm{~b}$ of their paper). If a different packing arrangement were used, then the cell problem discussed in section 3 would change accordingly, and a different homogenized model could arise.

Writing the diffusion-controlled cubic (101) in terms of the shrinking core timescale $\hat{t}$, and including the influence of the product gas, we obtain

$$
\frac{s(\hat{t})^{3}}{3}-\frac{s(\hat{t})^{2}}{2}+\frac{1}{6}=\frac{3 D_{e}}{4 \pi a^{3} \sigma} \frac{\gamma}{\beta} p_{a} \hat{t} .
$$

This is the same as the cubic for $l(t)$ in (121), up to the timescale, and hence our homogenized model gives the same behavior as the shrinking core model for diffusioncontrolled reactions. 
7.3. Comparison with the law of additive reaction times. Sohn [27] introduced the "law of additive reaction times." This states that, for a shrinking core reaction, the relationship between time and degree of conversion $X$ is the linear sum of the relationships in the diffusion-controlled limit and reaction-controlled limit. The ODE (20) in section 2 supports this law, since the diffusion limit $(\hat{\beta} \rightarrow \infty$ with $\hat{\beta} / \hat{\gamma}$ finite) and the reaction limit $(\hat{\beta} \rightarrow 0)$ combine to give the relationship between $l(t)$ and $t$. The total time for reaction in (21) is the sum of the total times in these limits (being $\hat{\beta} / 6 \hat{\gamma} p_{a}$ and $1 / \hat{\gamma} p_{a}$, respectively, for constant pressure $p_{a}$ ).

In section 5.2 we analyzed a homogenized model developed in section 3 , which allows for a diffuse reaction region. In Figure 9, we plotted the total time for reaction from the asymptotic limits of $\kappa \rightarrow \infty$ and $\kappa \rightarrow 0$, which correspond to the $\hat{\beta} \rightarrow \infty$ and $\hat{\beta} \rightarrow 0$ limits discussed above (namely, diffusion and reaction-controlled processes, respectively). We find that adding these reaction times together gives a good fit with numerics, adding further weight to Sohn's law.

7.4. Comparison with existing continuum models. Melchiori and collaborators (in $[18,19]$ ) develop continuum models and compare them with canonical shrinking core models. Their continuum models are of the form

$$
\epsilon_{M} \frac{\partial C_{A}}{\partial t}=D_{M} \nabla^{2} C_{A}-\sigma_{1} k_{M} \frac{C_{B}^{\alpha}}{C_{B 0}^{\alpha}} C_{A}, \quad \frac{\partial C_{B}}{\partial t}=-k_{M} \frac{C_{B}^{\alpha}}{C_{B 0}^{\alpha}} C_{A},
$$

where we have relabeled parameters $\epsilon_{M}, D_{M}$, and $k_{M}$ to avoid confusion with parameters in this paper; $C_{A}$ and $C_{B}$ are the molar concentrations of gas $A$ and solid $B$ (which we have relabeled to fit with notation in this paper), and $C_{B 0}$ is the initial concentration of solid $B$. The index $\alpha$ is a general power of the reaction. Since our $b(R, t)$ relates to their $C_{B}$ through $b^{3}=C_{B} / C_{B 0}$, then in the quasi-steady limit $\epsilon_{M} \rightarrow 0$, we obtain the model with (61) and (63) developed in section 3 and analyzed in section 5.2, but with $\alpha=2 / 3$ and $\lambda=0$. Melchiori and collaborators also note in $[18,19]$ that in the kinetic regime their continuum model gives the same results as a shrinking core model when $\alpha=2 / 3$, but that there are differences for any other reaction order. Thus our choice of a first order reaction rate (given in (7)), which corresponds to $\alpha=2 / 3$ in (125), allows this range of sharp interface shrinking core and spatially homogeneous continuum model behavior.

In addition, in our model, we have included the parameter $\lambda$, which allows for a range of behaviors across the diffusive boundary region in the diffusion-controlled reaction arising from the large $\kappa$ limit (see Figure 7). Our model also allows for a richer set of reaction behavior for general values of $\kappa$ (see Figure 5) and has the ability to better describe experimental results.

Szekely, Evans, and Sohn [33] developed a model for porous solids which takes the form

$$
\nabla^{2} p=\mu^{2} p b^{F_{g}-1}, \quad \frac{\partial b}{\partial t}=-p,
$$

where we have relabeled variables to avoid confusion with those used in this paper. The key difference from (61) and (63) with $\lambda=0$ is the "shape-factor" $F_{g}$, which is given by the dimension of the geometry of interest. For a one-dimensional slab $F_{g}=1$, for a cylinder $F_{g}=2$, and for a sphere $F_{g}=3$. This agrees with (63), which has been derived for a three-dimensional particle. Note that if we considered a one- or two-dimensional model, then we would replace $b^{3} / 3$ in (63) with $b$ or $b^{2} / 2$, respectively, as done in (126). In the case of a slab $\left(F_{g}=1\right)$, analytical solutions can be found to (126) without the need for asymptotics. 
8. Conclusions. We have derived a canonical shrinking core model that generalizes formulations in the literature, leading to a cubic relationship between the location of the interface and time. This model simplifies in diffusion-controlled and reactioncontrolled limits. This canonical shrinking core model has been used as a building block to develop homogenized models of gas-solid reactions which account for interaction between microscale granular behavior and macroscale particle dynamics. These are developed using the parameter $\delta$ as the ratio of the microscale to macroscale lengthscales, with $\mathcal{O}(1)$ and $\mathcal{O}\left(\delta^{2}\right)$ scalings being the resulting distinguished limits for the ratio of diffusivity in the grain to that in the pore space. More detailed analysis was dedicated to the $\mathcal{O}\left(\delta^{2}\right)$ distinguished limit, as it is the more physically relevant limit.

The homogenized model arising from the $\mathcal{O}\left(\delta^{2}\right)$ diffusion scaling agrees with models given by Melchiori et al. [18, 19] and Szekely, Evans, and Sohn [33], but with an additional parameter capturing the structure of the reaction given the concentration of the reactant solid. This parameter, $\lambda$, governs the shape of the reaction front in the diffusion-controlled process. A boundary layer of $\mathcal{O}\left(\kappa^{-1 / 2}\right)$ is found across which the solid varies from being fully reactant to fully product. The diffusion-controlled and reaction-controlled times for the reaction to be completed for both the shrinking core model in section $(2)$ and the $\mathcal{O}\left(\delta^{2}\right)$ homogeneous model agree with Sohn's law of additive reaction times. This gives support for the law that the time for a particle reaction is equal to the sum of the time when diffusion dominates and when the chemical reaction dominates.

Most of the modeling and analysis in this paper have been carried out under the assumption that the product gas has no influence on the reaction dynamics. However, in section 6 , we showed that this analysis can be extended to include the effects of the product gas through a parameter $\sigma$. This parameter depends on the diffusivity of the product gas, the cross-diffusivities of the two gases, and the stoichiometric coefficient describing how many moles of the product gas are produced per mole of the reactant gas reacted. This extension assumes that the binary gas system is related through a Fickian diffusion tensor. More accurate diffusion models could be employed, but at the cost of increasing the complexity of the analysis.

The model developed in this paper allows for a diffuse reaction front, which is an improvement on the sharp interface shrinking core model commonly used. In practice, the solid reactant used may be an agglomerated material, composed of many smaller grains compressed together by a binder material, suggesting that a diffusive reaction front is more appropriate. We have shown how the reaction can be limited by the chemical kinetics, diffusion through the granular solid product matrix, or diffusion through the intergranular void space or binder. The model captures the influence of gas concentration, solid concentration, particle size, grain size, kinetic rate, stoichiometry, and (binary) gas diffusivities on the reaction time, and also the dynamic transitions towards complete reaction. Thus this model for gas-solid reactions can be used in conjunction with other chemical reactions in process models, which typically consist of coupled reaction-advection-diffusion PDEs for chemical and thermal effects, and allow researchers in industry to simulate the influence of operational inputs on production levels.

In silicon production, a range of raw materials is used as the source of carbon, including petroleum coke, char, charcoal, wood chips, and agglomerated briquettes. Particle size, porosity, and chemical reactivity are all important considerations when selecting the material. For small particles of charcoal and coke, the canonical shrinking core model compares decently with experiments [20]. In such diffusion-controlled sit- 
uations, the shrinking core model gives similar behavior to our homogenized model. However, for other particles, such as large briquettes of compressed agglomerates, where diffusivity in the binder is important, the simple shrinking core approach is not a good representation, and our model is preferred. The role of binder during heat treatment of carbon has been studied in [23]. Since our model allows both sharp interface behavior and a diffuse reaction front, it is an ideal means of comparing the reactivity of different raw materials. We envisage similar benefits for other metallurgical and chemical processes.

In practice, the conversion of one solid to another through reaction with gas can lead to volumetric increase or decrease of grains within the particle, with the stresses caused by the solid expansion or contraction possibly causing cracks and fracturing of the solid (again see [23]). Such mechanical effects have not been considered in this paper, due to the difficulty in considering how displacements at the microscale grain level would influence neighboring grains inside a spherical particle. However, a study of the interactions with the chemical and diffusional effects considered in this paper could bring further valuable insight.

Appendix A. Small $\kappa$ limit equations. Here we list the systems arising at each of the first three orders in $\kappa$, with solutions given in (74) and (75). At $\mathcal{O}(1)$ we obtain the system

$$
\begin{gathered}
\frac{\partial^{2} W_{0}}{\partial R^{2}}=0, \quad b_{0}+\frac{\lambda}{2} b_{0}^{2}-\frac{\lambda}{3} b_{0}^{3}-\left(1+\frac{\lambda}{6}\right)=-\frac{1}{R} \int_{\tau=0}^{t} W_{0}(R, \tau) d \tau, \\
W_{0}(1, t)=1, \quad W_{0}=\mathcal{O}(R) \quad \text { as } R \rightarrow 0, \quad b_{0}(R, 0)=1 .
\end{gathered}
$$

At $\mathcal{O}(\kappa)$ we obtain

$$
\begin{gathered}
\frac{\partial^{2} W_{1}}{\partial R^{2}}=W_{0} b_{0}^{2}, \quad b_{1}+\lambda b_{0} b_{1}-\lambda b_{0}^{2} b_{1}=-\frac{1}{R} \int_{\tau=0}^{t} W_{1}(R, \tau) d \tau, \\
W_{1}(1, t)=0, \quad W_{1}=\mathcal{O}(R) \quad \text { as } R \rightarrow 0, \quad b_{1}(R, 0)=0,
\end{gathered}
$$

and at $\mathcal{O}\left(\kappa^{2}\right)$ we find the equations

$\frac{\partial^{2} W_{2}}{\partial R^{2}}=W_{1} b_{0}^{2}+2 W_{0} b_{0} b_{1}, \quad b_{2}+\frac{\lambda}{2} b_{1}^{2}+\lambda b_{0} b_{2}-\lambda b_{0}^{2} b_{2}-\lambda b_{0} b_{1}^{2}=-\frac{1}{R} \int_{\tau=0}^{t} W_{2}(R, \tau) d \tau$,

$$
W_{2}(1, t)=0, \quad W_{2}=\mathcal{O}(R) \quad \text { as } R \rightarrow 0, \quad b_{2}(R, 0)=0 .
$$

Appendix B. Influence of product gas equations. Here we list the equations and boundary conditions for $P^{V}, Q^{V}, P^{S}$, and $Q^{S}$. We obtain the system

$$
\begin{gathered}
0=\nabla_{\mathbf{y}}^{2} P^{V}+\delta\left(\nabla_{\mathbf{x}} \cdot \nabla_{\mathbf{y}} P^{V}+\nabla_{\mathbf{y}} \cdot \nabla_{\mathbf{x}} P^{V}\right)+\delta^{2} \nabla_{\mathbf{x}}^{2} P^{V}, \quad \mathbf{y} \in \omega_{V}, \\
0=\nabla_{\mathbf{y}}^{2} Q^{V}+\delta\left(\nabla_{\mathbf{x}} \cdot \nabla_{\mathbf{y}} Q^{V}+\nabla_{\mathbf{y}} \cdot \nabla_{\mathbf{x}} Q^{V}\right)+\delta^{2} \nabla_{\mathbf{x}}^{2} Q^{V}, \quad \mathbf{y} \in \omega_{V}, \\
0=\nabla_{\mathbf{y}}^{2} P^{S}+\delta\left(\nabla_{\mathbf{x}} \cdot \nabla_{\mathbf{y}} P^{S}+\nabla_{\mathbf{y}} \cdot \nabla_{\mathbf{x}} P^{S}\right)+\delta^{2} \nabla_{\mathbf{x}}^{2} P^{S}, \quad \mathbf{y} \in \omega_{S},
\end{gathered}
$$




$$
\begin{aligned}
& 0=\nabla_{\mathbf{y}}^{2} Q^{S}+\delta\left(\nabla_{\mathbf{x}} \cdot \nabla_{\mathbf{y}} Q^{S}+\nabla_{\mathbf{y}} \cdot \nabla_{\mathbf{x}} Q^{S}\right)+\delta^{2} \nabla_{\mathbf{x}}^{2} Q^{S}, \quad \mathbf{y} \in \omega_{S}, \\
& P^{V}=P^{S}, \quad Q^{V}=Q^{S} \quad \text { at } \mathbf{y}=a \mathbf{e}_{r}, \\
& \nabla_{\mathbf{y}} P^{V}+\delta \nabla_{\mathbf{x}} P^{V}=\delta^{n} \nu \nabla_{\mathbf{y}} P^{S}+\delta^{n+1} \nu \nabla_{\mathbf{x}} P^{S} \text { at } \mathbf{y}=a \mathbf{e}_{r}, \\
& \nabla_{\mathbf{y}} Q^{V}+\delta \nabla_{\mathbf{x}} Q^{V}=\delta^{n} \nu \nabla_{\mathbf{y}} Q^{S}+\delta^{n+1} \nu \nabla_{\mathbf{x}} Q^{S} \text { at } \mathbf{y}=a \mathbf{e}_{r}, \\
& -\nabla_{\mathbf{y}} P^{S} \cdot \mathbf{n}_{\mathbf{y}}+\delta\left(\nabla_{\mathbf{y}} P^{S} \cdot \nabla_{\mathbf{x}} b(\mathbf{x}, t)-\nabla_{\mathbf{x}} P^{S} \cdot \mathbf{n}_{\mathbf{y}}\right)+\delta^{2} \nabla_{\mathbf{x}} P^{S} \cdot \nabla_{\mathbf{x}} b(\mathbf{x}, t) \\
& =-\delta^{2-n} \frac{d_{3}+d_{1} \sigma_{2}}{d_{3}-d_{1} d_{2}} \frac{\beta}{\nu} P^{S}\left\|\mathbf{n}_{\mathbf{y}}-\delta \nabla_{x} b(\mathbf{x}, t)\right\| \text { at } \mathbf{y}=b(\mathbf{x}, t) \mathbf{e}_{r}, \\
& -\nabla_{\mathbf{y}} Q^{S} \cdot \mathbf{n}_{\mathbf{y}}+\delta\left(\nabla_{\mathbf{y}} Q^{S} \cdot \nabla_{\mathbf{x}} b(\mathbf{x}, t)-\nabla_{\mathbf{x}} Q^{S} \cdot \mathbf{n}_{\mathbf{y}}\right)+\delta^{2} \nabla_{\mathbf{x}} Q^{S} \cdot \nabla_{\mathbf{x}} b(\mathbf{x}, t) \\
& =\delta^{2-n} \frac{d_{2}+\sigma_{2}}{d_{3}-d_{1} d_{2}} \frac{\beta}{\nu} P^{S}\left\|\mathbf{n}_{\mathbf{y}}-\delta \nabla_{x} b(\mathbf{x}, t)\right\| \text { at } \mathbf{y}=b(\mathbf{x}, t) \mathbf{e}_{r},
\end{aligned}
$$

where we have obtained (140) and (141) from the first equation in (111) by premultiplying by the inverse of the diffusion matrix. We have that $P^{V}$ and $Q^{V}$ are periodic on $\partial \omega_{o}$, which is the outside boundary of the cell. The interface $b(\mathbf{x}, t)$ moves according to the equation

$$
\frac{\partial b(\mathbf{x}, t)}{\partial t}=-\gamma P^{S} \text { at } \mathbf{y}=b(\mathbf{x}, t) \mathbf{e}_{r},
$$

with initial condition $b(\mathbf{x}, 0)=a$. Hence the same system for $P$ in (32)-(36) holds, but with $\sigma \beta$ replacing $\beta$.

Acknowledgments. The authors thank A. Valderhaug, R. Birkeland, and H. Wegge (all at Elkem) for helpful discussions regarding this work. B. M. Sloman thanks Elkem for their financial support and the opportunity for on-site work during parts of this research.

\section{REFERENCES}

[1] H. Arunachalam, S. Onori, And I. Battiato, On veracity of macroscopic lithium-ion battery models, J. Electrochem. Soc., 162 (2015), pp. A1940-A1951.

[2] A. Bensoussan, J.-L. Lions, and G. Papanicolaou, Asymptotic Analysis for Periodic Structures, AMS, 2011.

[3] S. K. Bhatia and D. D. Perlmutter, A random pore model for fluid-solid reactions: I. Isothermal, kinetic control, AIChE J., 26 (1980), pp. 379-386.

[4] S. K. Bhatia and D. D. Perlmutter, A random pore model for fluid-solid reactions: II. Diffusion and transport effects, AIChE J., 27 (1981), pp. 247-254.

[5] M. Bruna and S. J. Chapman, Diffusion in spatially varying porous media, SIAM J. Appl. Math., 75 (2015), pp. 1648-1674, https://doi.org/10.1137/141001834.

[6] E. L. Cussler, Diffusion, Cambridge University Press, Cambridge, UK, 1997.

[7] M. P. Dalwadi, M. Bruna, and I. M. Griffiths, A multiscale method to calculate filter blockage, J. Fluid Mech., 809 (2016), pp. 264-289.

[8] M. P. DalWAdi, I. M. GRIFFIThS, AND M. BRunA, Understanding how porosity gradients can make a better filter using homogenization theory, Proc. R. Soc. A, 471 (2015), 20150464.

[9] S. Dargaville and T. W. Farrell, Predicting active material utilization in LiFePO4 electrodes using a multiscale mathematical model, J. Electrochem. Soc., 157 (2010), pp. A830A840.

Copyright $@$ by SIAM. Unauthorized reproduction of this article is prohibited. 
[10] S. Dargaville and T. W. Farrell, A comparison of mathematical models for phase-change in high-rate LiFePO4 cathodes, Electrochimica Acta, 111 (2013), pp. 474-490.

[11] M. Doyle, T. F. Fuller, And J. Newman, Modeling of galvanostatic charge and discharge of the lithium/polymer/insertion cell, J. Electrochem. Soc., 140 (1993), pp. 1526-1533.

[12] Y. Fu, Y. Jiang, S. Poizeau, A. Dutta, A. Mohanram, J. D. Pietras, and M. Z. Bazant, Multicomponent gas diffusion in porous electrodes, J. Electrochem. Soc., 162 (2015), pp. F613-F621.

[13] R. Gaur, L. Mishra, and S. K. Sen Gupta, Diffusion and transport of molecules in living cells, in Modelling and Simulation of Diffusive Processes, Springer, 2014, pp. 27-49.

[14] G. R. Gavalas, Analysis of char combustion including the effect of pore enlargement, Combust. Sci. Technol., 24 (1980), pp. 197-210.

[15] P. K. GBor AND C. Q. JiA, Critical evaluation of coupling particle size distribution with the shrinking core model, Chem. Engrg. Sci., 59 (2004), pp. 1979-1987.

[16] A. N. Gorban, H. P. Sargsyan, And H. A. WaHAB, Quasichemical models of multicomponent nonlinear diffusion, Math. Model. Natural Phenomena, 6 (2011), pp. 184-262.

[17] S. Homma, S. Ogata, J. Koga, and S. Матsumoto, Gas-solid reaction model for a shrinking spherical particle with unreacted shrinking core, Chem. Engrg. Sci., 60 (2005), pp. 49714980.

[18] T. Melchiori and P. CANu, Improving the quantitative description of reacting porous solids: Critical analysis of the shrinking core model by comparison to the generalized grain model, Indust. Engrg. Chem. Res., 53 (2013), pp. 8980-8995.

[19] T. Melchiori, F. Gallucci, M. van Sint Annaland, and P. Canu, Reacting porous solids with phase segregation, Chem. Engrg. Sci., 132 (2015), pp. 200-214.

[20] E. Myrhaug, J. Tuset, and H. Tveit, Reaction mechanisms of charcoal and coke in the silicon process, in Proceedings: Tenth International Ferroalloys Congress, Vol. 1, 2004, pp. 108-121.

[21] J. Y. PARK AND O. LeVenspiel, The crackling core model for the reaction of solid particles, Chem. Engrg. Sci., 30 (1975), pp. 1207-1214.

[22] G. Pavliotis and A. Stuart, Multiscale Methods: Averaging and Homogenization, Springer Science \& Business Media, 2008.

[23] L. Roberts, E. Nordgånd-Hansen, Ø. Mikkelsen, S. A. Halvorsen, and R. A. Van GORDER, $A$ heat and mass transfer study of carbon paste baking, Internat. Commun. Heat Mass Transfer, 88 (2017), pp. 9-19.

[24] A. Schei, J. K. Tuset, And H. Tveit, Production of High Silicon Alloys, Tapir, Trondheim, Norway, 1998.

[25] B. M. Sloman, C. P. Please, R. A. Van Gorder, A. M. Valderhaug, R. G. Birkeland, AND H. WegGe, A heat and mass transfer model of a silicon pilot furnace, Metallurg. Materials Trans. B, 48 (2017), pp. 2664-2676.

[26] R. B. Smith And M. Z. BAZAnt, Multiphase porous electrode theory, J. Electrochem. Soc., 164 (2017), pp. E3291-E3310.

[27] H. Y. SoHn, The law of additive reaction times in fluid-solid reactions, Metallurg. Materials Trans. B, 9 (1978), pp. 89-96.

[28] H. Y. SoHN, Chemical reaction engineering in the chemical processing of metals and inorganic materials, part I. Advances in fluid-solid reaction analysis, Korean J. Chem. Engrg., 20 (2003), pp. 185-199.

[29] H. Y. SOHN AND J. SzEKELY, A structural model for gas-solid reactions with a moving boundaryIII: A general dimensionless representation of the irreversible reaction between a porous solid and a reactant gas, Chem. Engrg. Sci., 27 (1972), pp. 763-778.

[30] V. SRinivasan and J. Newman, Discharge model for the lithium iron-phosphate electrode, J. Electrochem. Soc., 151 (2004), pp. A1517-A1529.

[31] J. Szekely ANd J. W. Evans, A structural model for gas-solid reactions with a moving boundary, Chem. Engrg. Sci., 25 (1970), pp. 1091-1107.

[32] J. Szekely And J. W. Evans, A structural model for gas-solid reactions with a moving boundary-II: The effect of grain size, porosity and temperature on the reaction of porous pellets, Chem. Engrg. Sci., 26 (1971), pp. 1901-1913.

[33] J. Szekely, J. W. Evans, And H. Y. Sohn, Gas-Solid Reactions, Academic Press, New York, 1976.

[34] C. Y. Wen, Noncatalytic heterogeneous solid-fluid reaction models, Indust. Engrg. Chem., 60 (1968), pp. 34-54.

[35] Z. Xu, X. Sun, And M. A. Khaleel, A generalized kinetic model for heterogeneous gas-solid reactions, J. Chem. Phys., 137 (2012), 074702.

[36] S. YAGi AND D. Kuni, Studies on combustion of carbon particles in flames and fluidized beds, Symposium (Intern.) Combustion, 5 (1955), pp. 231-244.

Copyright $@$ by SIAM. Unauthorized reproduction of this article is prohibited. 